Proyecciones

Vol. 26, $\mathrm{N}^{o}$ 1, pp. 37-71, May 2007.

Universidad Católica del Norte

Antofagasta - Chile

\title{
ABOUT DECAY OF SOLUTION OF THE WAVE EQUATION WITH DISSIPATION *
}

\author{
LUIS CORTÉS \\ UNIVERSIDAD DE ANTOFAGASTA, CHILE \\ and \\ YOLANDA SANTIAGO \\ UNIVERSIDAD NACIONAL MAYOR DE SAN MARCOS, PERÚ
}

Received : November 2006. Accepted : March 2007

\begin{abstract}
In this work, we consider the problem of existence of global solutions for a scalar wave equation with dissipation. We also study the asymptotic behaviour in time of the solutions. The method used here is based in nonlinear techniques.

Key words: wave equation, evolution model, decay of solution, asymptotic behaviour.
\end{abstract}

${ }^{*}$ Support: see acknowledgements. 


\section{Introduction}

We will study the following evolution problem

$$
\begin{aligned}
u_{t t}-\Delta u+a(x) u_{t}= & 0 \text { in } \Omega \times \mathbf{R}^{+}, \\
u= & 0 \text { on }, \partial \Omega \times \mathbf{R}^{+}, \\
u(0)=u^{0}, & u_{t}(0)=u^{1} .
\end{aligned}
$$

where $\Omega$ is an open bounded domain in $\mathbf{R}^{N}$ with smooth boundary $\partial \Omega$ and $a$ is a suitable, smooth and should not identically zero on $\bar{\Omega}$; besides, $a$ can vanish in some part of $\bar{\Omega}$.

We define by

$$
E(t)=\frac{1}{2} \int_{\Omega}\left|u_{t}\right|^{2}+|\nabla u|^{2} d x
$$

the energy associated to the system (1.1)-(1.3). By Lemma $5.1 E$ is non increasing. Thus, we are interested in finding out what happens to $E(t)$ as $t$ goes to infinity and what is its rate of decay.

In this work, we study the existence of global solution and the asymptotic behaviour of the wave equation with dissipation, where the initial conditions satisfy the $m$ th-order compatibility condition, with allows us to obtain a more regular solution.

We use the semigroup theory [17], [6] to prove the existence and uniqueness of solution to the problem (1.1)-(1.3), as well as its continuous dependency of initial data. Likewise, we study the regularity of this solution.

In section 4 we make a complete study of certain integral inequalities [15]. Also we prove that

$$
\int_{t}^{\infty} f(\tau)^{1+\tau} d \tau \leq C F(t) \text { implies } f(t) \leq \frac{C}{(1+t)^{\frac{1}{\sigma}}}
$$

and we use it in the proof of Lemma 4.2. Besides, in this section we introduce Lemma 4.4 which is an analogous version of Lemma 4.3.

Making use of the multiplicative techniques [13], we obtain important estimations like (5.12), (5.16), (5.23). And by adapting the Conrad and Rao methods [1], we obtain the estimation (5.44) which allow us to prove Lemma 5.3 and then the hypothesis of the Lemma 4.4.

Another study can be seen in Nakao [16]. We are strongly motive by the most challenging mathematical results have already been obtained in related topics, see for instance $[9,10,11,12],[7,8][18],[19],[20],[21]$ and $[14,22]$, among others. 


\section{Main Results}

We state the result for existence of solution to the problem (1.1)-(1.3).

Theorem 2.1. Given $\left(u_{0}, u_{1}\right) \in\left(H^{2}(\Omega) \cap H_{0}^{1}(\Omega)\right) \times H_{0}^{1}(\Omega)$, there is only one solution $u(x, t)$ of $(1.1)$ - (1.3) in $C^{2}\left([0, \infty), L^{2}(\Omega)\right) \cap C^{1}\left([0, \infty), H_{0}^{1}(\Omega)\right) \cap$ $C\left([0, \infty), H^{2}(\Omega) \cap H_{0}^{1}(\Omega)\right)$.

Also, we will need the following result for regularity of the solution, for which we cite Kesavan [6] and Ikawa [5]. We introduce the following definition

Definition 2.1. The initial condition $\left(u^{0}, u^{1}\right) \in H^{m+1} \times H^{m}$ satisfies the mth - order compatibility condition associated to (1.1)-(1.3) if

$$
u^{k} \in H^{m+1-k} \cap H_{0}^{1} \text { for } k=0,1, \ldots, m \text { and } u^{m+1} \in L^{2},
$$

where the sequence $\left(u^{k}\right)_{k}$ is defined by induction from $\left(u^{0}, u^{1}\right)$ by the formula

$$
u^{k+2}=\Delta u^{k}-a(x) u^{k+1}
$$

Proposition 2.1. Let $m \geq 1$ be an integer. Let us suppose that $a \in$ $C^{m-1}(\bar{\Omega})$ and $\left(u^{0}, u^{1}\right)$ satisfies the $m$ th- order compatibility condition associated to (1.1)- (1.3). Then, there is only one solution $u(t)$ of the problem (1.1)-(1.3) such that

$$
u \in X_{m}=\bigcap_{k=0}^{m} C^{k}\left(\mathbf{R}^{+}, H^{m+1-k} \cap H_{0}^{1}\right) \cap C^{m+1}\left(\mathbf{R}^{+}, L^{2}\right),
$$

and the linear application

$$
\left(u^{0}, u^{1}\right) \in H^{m+1}(\Omega) \times H^{m}(\Omega) \longrightarrow u \in X_{m}
$$

is continuous. That is exists $C>0$ such that $\sum_{k=0}^{m+1}\left\|D^{k} u(t)\right\|_{L^{2}}^{2} \leq C\left\|\left(u^{0}, u^{1}\right)\right\|_{H^{m+1} \times H^{m}}^{2}$, where $D^{k}$ denotes any kth order partial differentiation with respect to $t$ and $x$.

Let us suppose that $\Omega$ is the open ball in $\mathbf{R}^{N}$ centered on 0 and the radius $R$. Let us also assume that $\forall|x| \geq \frac{R}{2}, \quad a(x):=\tilde{a}(|x|)$, where 
$\tilde{a}:\left[\frac{R}{2}, R\right] \rightarrow \mathbf{R}^{+}$is a strictly decreasing function which satisfies $\tilde{a}(R)=0$. (Note that $\frac{R}{2}$ could be replaced by any $R-\epsilon$ with $\epsilon>0$ ). Set

$$
\forall r \in\left[0, \frac{R}{2}\right], \quad b(r):=\tilde{a}(R-r) \text { and } B(r):=r b(r) .
$$

We observe that $B$ is continuous and strictly increasing on $\left[0, \frac{R}{2}\right]$ and that $B(0)=0$. Also,

$$
b(r) \rightarrow 0=\tilde{a}(R) \text { as } r \rightarrow 0
$$

That is

$$
a(x) \rightarrow 0 \text { as } x \rightarrow \partial \Omega .
$$

We will use the Lemma 4.4 in the proof the following Main Theorem.

Theorem 2.2 (Main result). Let us suppose that a goes to zero at the boundary quickly enough so that there exist $p>0$ and $C>0$ such that

$$
\forall \rho \in\left(0, \frac{R}{2}\right), \quad \int_{\rho}^{\frac{R}{2}} \frac{1}{b(r)^{p}} d r \leq C \frac{\rho}{b(\rho)^{p}} .
$$

Set $m>\frac{N}{2}$. Then, if $\left(u^{0}, u^{1}\right)$ satisfies the $m$ th-order compatibility condition; there exists $C>0$ which depends on the norm of the initial condition on $H^{m+1}(\Omega) \times H^{m}(\Omega)$ such that the solution $u$ of (1.1)-(1.3) verifies

$$
E(t) \leq C\left(B^{-1}\left(\frac{1}{t}\right)\right)^{\frac{2 m}{N}}
$$

where $B^{-1}$ denotes the inverse function of $B$.

\subsection{Remarks of Theorem}

Remark 2.1. If exists $n$ in $\mathbf{N}$ such that $n \geq 2$ and exists $p>0$ such that $b(r) \leq n r^{\frac{1}{p}} b(n r)$, for all $r \in\left[0, \frac{R}{2 n}\right]$, then (2.6) holds.

Remark 2.2. If $b(r)=r^{k}$ with $k>0$ and $p k>1$, then (2.6) holds.

Therefore, by the main theorem we obtain

$$
E(t) \leq \frac{C}{t^{\frac{1}{(k+1) \theta}}} .
$$

Remark 2.3. If $b(r)=\frac{1}{|L n r|}$ then (2.6) is not true. 
Remark 2.4. If $b(r)=r^{q} e^{-\frac{1}{r^{k}}}$ with $k>0$ then, we can apply remark 2.1. Therefore, by the main theorem we get

$$
E(t) \leq \frac{C}{\left[(\operatorname{Ln} t)^{\frac{1}{k}}\right]^{\frac{2 m}{N}}} .
$$

\section{Existence of solution}

From the equation (1.1) writing $v=u_{t}$ we get:

$$
\left(\begin{array}{l}
u \\
v
\end{array}\right)_{t}=\left(\begin{array}{c}
u_{t} \\
u_{t t}
\end{array}\right)=\left(\begin{array}{c}
u_{t} \\
\Delta u-a u_{t}
\end{array}\right)=\left(\begin{array}{c}
v \\
\Delta u-a v
\end{array}\right)=\left(\begin{array}{cc}
0 & I \\
\Delta & -a I
\end{array}\right)\left(\begin{array}{l}
u \\
v
\end{array}\right)
$$

we define the Operator $A: D(A) \subset H \longrightarrow H$,

$$
A=\left(\begin{array}{cc}
0 & I \\
\Delta & -a I
\end{array}\right)
$$

where $H=H_{0}^{1}(\Omega) \times L^{2}(\Omega)$ and $D(A)=H^{2}(\Omega) \cap H_{0}^{1}(\Omega) \times H_{0}^{1}(\Omega)$.

Thus (1.1)-(1.3) is equivalent to

$$
\text { I.V.P }\left\{\begin{array}{l}
U_{t}(t)=A U(t) \\
U(0)=U_{0}:=\left(\begin{array}{l}
u_{0} \\
u_{1}
\end{array}\right) \in D(A) .
\end{array}\right.
$$

Theorem 3.1. The Operator $A$ defined above generates a contraction semigroup $S(t)$ on the Hilbert Space $H$.

Proof.- Observe that $D(A)$ is dense in $H$. We will prove that $A$ is dissipative. Let $U=(u, v)^{T} \in D(A)$ then

$$
\begin{aligned}
<A U, U> & =\sum_{i=1}^{N} \int_{\Omega} \frac{\partial v}{\partial x_{i}} \frac{\overline{\partial u}}{\partial x_{i}} d x+\int_{\Omega}(\Delta u-a(x) v) \bar{v} d x \\
& =\sum_{i=1}^{N} \int_{\Omega} \frac{\partial v}{\partial x_{i}} \frac{\overline{\partial u}}{\partial x_{i}} d x+\int_{\Omega} \Delta u \bar{v}-a(x)|v|^{2} d x \\
& =\sum_{i=1}^{N} \int_{\Omega} \frac{\partial v}{\partial x_{i}} \frac{\overline{\partial u}}{\partial x_{i}}-\frac{\partial u}{\partial x_{i}} \frac{\partial \bar{v}}{\partial x_{i}} d x-\int_{\Omega} a(x)|v|^{2} d x \\
=- & \sum_{i=1}^{N} \int_{\Omega} 2 i \operatorname{Im}\left(\frac{\partial u}{\partial x_{i}} \frac{\partial \bar{v}}{\partial x_{i}}\right) d x-\int_{\Omega} a(x)|v|^{2} d x,
\end{aligned}
$$


where $\operatorname{Im}(z)$ is the imaginary part of $z \in \mathbf{C}$. Taking the real part of the equality (3.2), we have

$$
\operatorname{Re}\left(<A U, U>_{H_{0}^{1}(\Omega) \times L^{2}(\Omega)}\right)=-\int_{\Omega} a(x)|v|^{2} d x \leq 0
$$

Now, we will prove that $0 \in \rho(A)$. In fact, let $F=(f, g)^{T} \in H_{0}^{1}(\Omega) \times$ $L^{2}(\Omega)=H$, we will prove that there is $U=(u, v)^{T} \in D(A)$, such that $A U=F$. Let us consider the equations

$$
\begin{aligned}
v & =f \in H_{0}^{1}(\Omega) \\
\Delta u-a(x) v & =g \in L^{2}(\Omega) .
\end{aligned}
$$

Replacing (3.3) in (3.4), we have

$$
\Delta u=a(x) f+g \in L^{2}(\Omega) .
$$

By standard results on Elliptic linear equations, we have that (3.5) has only one solution $u \in H^{2}(\Omega) \cap H_{0}^{1}(\Omega)$. From (3.3) we obtain $v=f$. That is, $A$ is an onto map.

We claim that $A$ is one to one. In fact, let $A U=0$ then

$$
\begin{aligned}
v & =0 \\
\Delta u-a(x) v & =0 .
\end{aligned}
$$

Replacing (3.6) in (3.7) we have $\Delta u=0$ and using the Green's Identity we have

$$
|u|_{H_{0}^{1}(\Omega)}^{2}=\int_{\Omega}|\nabla u|^{2} d x=\int_{\Omega} \Delta u \bar{u} d x=0
$$

hence $u=0$ in $H_{0}^{1}(\Omega)$. From (3.6) we have that $v=0$. Therefore $U=0$. i.e. $A$ is one to one.

Thus, there is $A^{-1}: H \longrightarrow D(A)$ because $A$ is one to one and $H$ is the image of $A$. Now, we will prove that $A^{-1}$ is bounded. Multiplying the equation (3.5) by $\bar{u}$ and integrating on $\Omega$, we have

$$
\int_{\Omega} \Delta u \bar{u} d x=\int_{\Omega}(a f+g) \bar{u} d x
$$

but since $\int_{\Omega}|\nabla u|^{2} d x=\int_{\Omega} \Delta u \bar{u} d x$, using the Holder and Poincaré inequalities, we obtain

$$
\int_{\Omega}|\nabla u|^{2} d x=\int_{\Omega}(a f+g) \bar{u} d x \leq|u|_{L^{2}}|a f+g|_{L^{2}}
$$




$$
\begin{aligned}
& \leq \epsilon \int_{\Omega}|u|^{2} d x+C(\epsilon) \int_{\Omega}|a f+g|^{2} d x \\
& \leq \epsilon C_{p} \int_{\Omega}|\nabla u|^{2} d x+C(\epsilon) \int_{\Omega}|a f+g|^{2} d x .
\end{aligned}
$$

Then, taking $\epsilon>0$ such that $1-\epsilon C_{p}>0$ we have

$$
\left(1-\epsilon C_{p}\right) \int_{\Omega}|\nabla u|^{2} d x \leq C(\epsilon) \int_{\Omega}|a f+g|^{2} d x,
$$

that is

$$
\sqrt{1-\epsilon C_{p}}|\nabla u|_{L^{2}} \leq \sqrt{C(\epsilon)}|a f+g|_{L^{2}}
$$

Hence we have

$$
|\nabla u|_{L^{2}} \leq \frac{\sqrt{C(\epsilon)}}{\sqrt{1-\epsilon C_{p}}}|a f+g|_{L^{2}} \leq \frac{\sqrt{C(\epsilon)}}{\sqrt{1-\epsilon C_{p}}}\left\{|a|_{\infty}|f|_{L^{2}}+|g|_{L^{2}}\right\}
$$

Thus, using (3.8), $v=f$, and the Holder and Poincaré inequalities we get

$$
\begin{aligned}
|U|_{H}=|\nabla u|_{L^{2}}+|v|_{L^{2}}=|\nabla u|_{L^{2}}+|f|_{L^{2}} & \leq \hat{C}\left\{|f|_{L^{2}}+|g|_{L^{2}}\right\} \\
& \leq \hat{\hat{C}}\left\{|\nabla f|_{L^{2}}+|g|_{L^{2}}\right\} .
\end{aligned}
$$

Then,

$$
|U|_{H} \leq \hat{\hat{C}}|A U|_{H},
$$

that is

$$
\left|A^{-1} F\right|_{H} \leq \hat{\hat{C}}|F|_{H},
$$

which allow us to say that $A^{-1}$ is bounded. Now, by the Lummer-Phillips theorem, we have that $A$ is the infinitesimal generator of a $C_{0}$ semigroup of contraction on $H: S(t)$.

Remark 3.1. By Theorem 4.3.2 in [6], if $D(A) \ni U$ then $S(t) U \in C^{1}([0, \infty), H) \cap C([0, \infty), D(A))$.

Remark 3.2. By Remark 4.3.3 in [6], $U(t):=S(t) U_{0}$ is the solution of IVP (3.1) and it is the unique.

From these two remarks, we get the following result.

Proposition 3.1. There exists only one solution of (3.1),

$U(t) \in C^{1}\left([0, \infty), H_{0}^{1}(\Omega) \times L^{2}(\Omega)\right) \cap C\left([0, \infty),\left(H^{2}(\Omega) \cap H_{0}^{1}(\Omega)\right) \times H_{0}^{1}(\Omega)\right)$. 
Now, we will finish the proof of Theorem 2.1

Since $U_{0}=\left(u^{0}, u^{1}\right) \in D(A)$, by Proposition 3.1 we obtain that there exists $U(t) \in C^{1}\left([0, \infty), H_{0}^{1}(\Omega) \times L^{2}(\Omega)\right) \cap C\left([0, \infty),\left(H^{2}(\Omega) \cap H_{0}^{1}(\Omega)\right) \times\right.$ $H_{0}^{1}(\Omega)$ ) solution of (3.1) such that $U(0)=U_{0}, U(t) \in D(A), \forall t \in \mathbf{R}^{+}$.

Since $U$ satisfies (3.1) we have $u_{t}=v$ and $v_{t}=\Delta u-a v$. By one hand, we have $u_{t} \in C^{0}\left(\mathbb{R}^{+}, H_{0}^{1}(\Omega)\right)$, but since $u \in C^{0}\left(\mathbf{R}^{+}, H_{0}^{1}(\Omega)\right)$ then $u \in C^{1}\left(\mathbf{R}^{+}, H_{0}^{1}(\Omega)\right)$. Also, $u_{t t}=v_{t}=\Delta u-a u_{t} \in C\left(\mathbf{R}^{+}, L^{2}(\Omega)\right)$, but $u_{t}$ and $u$ belong to $C\left(\mathbf{R}^{+}, L^{2}(\Omega)\right)$ then $u \in C^{2}\left(\mathbf{R}^{+}, L^{2}(\Omega)\right)$. We also obtain that $u \in C\left(\mathbf{R}^{+}, H^{2}(\Omega) \cap H_{0}^{1}(\Omega)\right)$.

Remark 3.3. By the Hille-Yosida Theorem (Theorem 4.4.3 in [6] ), since A is the infinitesimal generator of contraction semigroup, $A$ is closed, $D(A)$ is dense in $H$ and $\forall \lambda>0, \exists(\lambda I-A)^{-1}$ bounded, moreover $\left\|(\lambda I-A)^{-1}\right\| \leq \frac{1}{\lambda}$.

Remark 3.4. Since $A$ is closed and there exists $A^{-1}$ (It was proved in $0 \in \rho(A))$, then $A^{-1}$ is also closed.

\section{Integral Inequalities}

Lemma 4.1. Let $E: \mathbf{R}^{+} \rightarrow \mathbf{R}^{+}$be a no increasing function and $\phi: \mathbf{R}^{+} \rightarrow$ $\mathbf{R}^{+}$be an strictly increasing $C^{1}$ function such that

$$
\phi(0)=0 \quad \text { and } \quad \phi(t) \longrightarrow+\infty \text { as } t \rightarrow+\infty .
$$

Let us suppose that there are $\sigma \geq 0$, and $w>0$ such that

$$
\forall s \geq 0, \quad \int_{s}^{+\infty} E(t)^{1+\sigma} \phi^{\prime}(t) d t \leq \frac{1}{w} E(0)^{\sigma} E(s) .
$$

Then, E satisfies the following estimates:

$$
\begin{gathered}
\text { If } \sigma=0 \text {, then } E(t) \leq E(0) e^{1-w \phi(t)}, \quad \text { for all } t \geq 0 \text {. } \\
\text { If } \sigma>0 \text {, then } E(t) \leq E(0)\left(\frac{1+\sigma}{1+w \sigma \phi(t)}\right)^{\frac{1}{\sigma}}, \quad \forall t \geq 0 .
\end{gathered}
$$

Proof.- Is enough to prove the case $E(0)=1$, because if $1 \neq E(0)=d>0$, we define $F(t):=\frac{E(t)}{E(0)}$, then $F(0)=1$ and applying (4.2) to $E(t)$ we have

$$
\begin{aligned}
\int_{s}^{\infty} F(t)^{(1+\sigma)} \phi^{\prime}(t) d t & =\frac{1}{E(0)^{1+\sigma}} \int_{s}^{+\infty} E(t)^{(1+\sigma)} \phi^{\prime}(t) d t \\
& \leq \frac{1}{w} E(0)^{\sigma} E(s) \frac{1}{E(0)^{(1+\sigma)}} \\
& =\frac{1}{w} \frac{E(s)}{E(0)}=\frac{1}{w} F(s)
\end{aligned}
$$


i.e. (4.2) holds for $F(s)$. Then

$$
\begin{aligned}
& \text { If } \sigma=0, \text { then } F(t) \leq e^{1-w \phi(t)}, \quad \forall t \geq 0, \\
& \text { i.e. } E(t) \leq E(0) e^{1-w \phi(t)}, \quad \forall t \geq 0 . \\
& \text { If } \sigma>0 \text {, then } F(t) \leq\left(\frac{1+\sigma}{1+w \sigma \phi(t)}\right)^{\frac{1}{\sigma}}, \quad \forall t \geq 0, \\
& \text { i.e. } E(t) \leq E(0)\left(\frac{1+\sigma}{1+w \sigma \phi(t)}\right)^{\frac{1}{\sigma}}, \quad \forall t \geq 0 .
\end{aligned}
$$

Now, we prove for $E(0)=1$.

We introduce the following function $f: \mathbb{R}^{+} \longrightarrow \mathbb{R}^{+}$defined by

$$
f(\tau):=E\left(\phi^{-1}(\tau)\right)
$$

then $f$ is no increasing. Making a change of variable and using (4.2) with $E(0)=1$, we obtain the following: $\forall 0<S<T<\infty$,

$$
\begin{aligned}
\int_{\phi(S)}^{\phi(T)} f(\tau)^{(1+\sigma)} d \tau & =\int_{\phi(S)}^{\phi(T)} E\left(\phi^{-1}(\tau)\right)^{(1+\sigma)} d \tau \\
& =\int_{S}^{T} E(t)^{(1+\sigma)} \phi^{\prime}(t) d t \\
& \leq \frac{1}{w} E(S)=\frac{1}{w} f(\phi(S)) .
\end{aligned}
$$

Since $\lim _{T \rightarrow \infty} \phi(T)=+\infty$, then $f$ satisfies:

$$
\forall S \geq 0, \quad \int_{S}^{+\infty} f(\tau)^{1+\sigma} d \tau \leq \frac{1}{w} f(S)
$$

Let us denote $h: \mathbf{R}^{+} \longrightarrow \mathbf{R}^{+}, h(t):=\int_{t}^{+\infty} f(\tau)^{(1+\sigma)} d \tau$. So, $h$ is well defined, no increasing, no negative and satisfies the following differential inequality.

$$
\forall t \geq 0 \quad-h^{\prime} \geq(w h)^{1+\sigma} .
$$

In fact, from $h(t)=-\int_{+\infty}^{t} f(\tau)^{(1+\sigma)} d \tau>0$ we have $h^{\prime}(t)=-f(t)^{(1+\sigma)}<0$. And so, using (4.5) we have that $-h^{\prime}(t)=f(t)^{(1+\sigma)} \geq(w h(t))^{(1+\sigma)}$.

Let us define $T_{0}:=\sup \{t: h(t)>0\}$. Then, if $\sigma=0, h$ satisfies:

$$
\forall 0 \leq t<T_{0}, \quad h(t) \leq h(0) e^{-w t} \leq \frac{1}{w} e^{-w t} .
$$


In fact, from (4.6) with $\sigma=0$ we have $-h^{\prime} \geq w h$, that is $h^{\prime}+w h \leq 0$, then $\left[e^{-w t} h\right]^{\prime} \leq 0$, from where $e^{w t} h(t) \leq h(0)$ holds.

By other hand, from (4.5) and $f(0)=E(0)=1$, we have that $h(0) w \leq$ $f(0)=1$, that is $h(0) \leq \frac{1}{w}$. Therefore, $h(t) \leq \frac{1}{w} e^{-w t}$. We observe that the estimate (4.7) holds if $t \geq T_{0}$. In fact, if $t \geq T_{0}$ then $h(t) \leq 0<\frac{1}{w} e^{-w t}$.

Let $\epsilon>0$. Since $f$ is no increasing, we have that

$$
\forall t \geq \epsilon, \quad f(t) \leq \frac{1}{\epsilon} \int_{t-\epsilon}^{t} f(\tau) d \tau \leq \frac{1}{\epsilon} h(t-\epsilon) \leq \frac{1}{w \epsilon} e^{w \epsilon} e^{-w t} .
$$

In fact, in the last inequality of (4.8) it is used (4.7).

On the other hand, since $f$ is no increasing, we have that $f(t) . \epsilon \leq$ $\int_{t-\epsilon}^{t} f(\tau) d \tau$. Also $\int_{t-\epsilon}^{t} f(\tau) d \tau \leq \int_{t-\epsilon}^{+\infty} f(\tau) d \tau=h(t-\epsilon)$.

If we take $\epsilon=\frac{1}{w}$ in (4.8), we get

$$
\forall t \geq \frac{1}{w}, \quad f(t) \leq e^{1-w t} .
$$

Since $E(t)=f(\phi(t))$, by using (4.9), we get (4.3).

If $\sigma>0, h$ satisfies:

$$
\text { for all } t \in\left[0, T_{0}\left[, \quad\left(h^{-\sigma}\right)^{\prime} \geq \sigma w^{(1+\sigma)} .\right.\right.
$$

In fact, $\left(h^{-\sigma}\right)^{\prime}=-\sigma h^{-(1+\sigma)} \cdot h^{\prime}=-\sigma h^{-(1+\sigma)} \cdot\left(-f^{1+\sigma}\right)=\sigma h^{-(1+\sigma)} f^{1+\sigma}=$ $\sigma\left(\frac{f}{h}\right)^{1+\sigma}$. But from (4.5) we have that $w \leq \frac{f}{h}$, from here $w^{1+\sigma} \leq\left(\frac{f}{h}\right)^{1+\sigma}$ then the result holds.

Integrating the inequality (4.10) from 0 to $t$, we obtain

$$
[h(t)]^{-\sigma}-\left[h(0]^{-\sigma} \geq \sigma w^{1+\sigma} t \quad \forall 0 \leq t<T_{0},\right.
$$

Thus

$$
h(t) \leq\left(h(0)^{-\sigma}+\sigma w^{1+\sigma} t\right)^{-\frac{1}{\sigma}}, \quad \forall 0 \leq t<T_{0} .
$$

Since $f$ is no increasing, we have for all $s \geq 0$

$$
(4.12)\left(\frac{1}{w}+\sigma s\right) f\left(\frac{1}{w}+(\sigma+1) s\right)^{\sigma+1} \leq \int_{s}^{\frac{1}{w}+(\sigma+1) s} f(\tau)^{\sigma+1} d \tau \leq h(s) .
$$

By the other hand, from (4.5) we have that $h(0) \leq \frac{1}{w} f(0)=\frac{1}{w} E(0)=\frac{1}{w}$, that is $w^{\sigma} \leq \frac{1}{h(0)^{\sigma}}$, then

$$
\left(h(0)^{-\sigma}+\sigma w^{1+\sigma} t\right)^{-\frac{1}{\sigma}} \leq\left(w^{\sigma}+\sigma w^{1+\sigma} t\right)^{-\frac{1}{\sigma}}=\frac{1}{w[1+\sigma w s]^{\frac{1}{\sigma}}} .
$$


Using (4.11), (4.12) and (4.13) we have

$$
\left(\frac{1+w \sigma s}{w}\right) f\left(\frac{1}{w}+(\sigma+1) s\right)^{\sigma+1} \leq \frac{1}{w[1+\sigma w s]^{\frac{1}{\sigma}}},
$$

hence

$$
\forall s \geq 0, \quad f\left(\frac{1}{w}+(\sigma+1) s\right) \leq \frac{1}{(1+w \sigma s)^{\frac{1}{\sigma}}} .
$$

Putting $t=\frac{1}{w}+(\sigma+1) s$ on (4.14), we get

$$
f(t) \leq\left(\frac{1+\sigma}{1+w \sigma t}\right)^{\frac{1}{\sigma}}, \forall t \geq 0 .
$$

Finally, since $E(t)=f(\phi(t))$, using (4.15) we get (4.4).

From (4.4) we deduce the following result:

Corollary 4.1. Let $f: \mathbf{R}^{+} \rightarrow \mathbf{R}^{+}$be a no increasing and continuous function. Let us assume that there are $\sigma>0, \sigma^{\prime}>0$, and $c>0$ such that

$$
\forall t \geq 0, \quad \int_{t}^{+\infty} f(\tau)^{1+\sigma} d \tau \leq c \frac{f(0)^{\sigma} f(t)}{(1+t)^{\sigma^{\prime}}} .
$$

Then, there exists $C>0$ such that,

$$
\forall t>0, \quad f(t) \leq f(0) \frac{C}{(1+t)^{\frac{\left(1+\sigma^{\prime}\right)}{\sigma}}} .
$$

Proof.- Is enough to prove the case $f(0)=1$, because if $1 \neq f(0)=d>0$ we define $g(s):=\frac{f(s)}{f(0)}$, then $g(0)=1$ and

$$
\begin{aligned}
\int_{t}^{+\infty} g(s)^{1+\sigma} d s & =\frac{1}{f(0)^{(1+\sigma)}} \int_{t}^{+\infty} f(s)^{(1+\sigma)} d s \\
& \leq \frac{1}{f(0)^{\sigma} f(0)} c \frac{f(0)^{\sigma} f(t)}{(1+t)^{\sigma^{\prime}}} \\
& =c g(0)^{\sigma} \frac{g(t)}{(1+t)^{\sigma^{\prime}}}
\end{aligned}
$$

that is (4.16) holds. Using (4.17) for $g(0)=1$ we have

$$
g(t) \leq g(0) \frac{C}{(1+t)^{\frac{\left(1+\sigma^{\prime}\right)}{\sigma}}},
$$


that is,

$$
f(t) \leq \frac{C f(0)}{(1+t)^{\frac{\left(1+\sigma^{\prime}\right)}{\sigma}}} .
$$

Now, we prove for $f(0)=1$.

If $t \geq 0$, let us define

$$
g(t)=\frac{f(t)}{(1+t)^{\sigma^{\prime}}}
$$

then $g$ is no increasing. Since $g(\tau)^{1+\sigma}(1+\tau)^{\sigma^{\prime}(1+\sigma)}=f(\tau)^{1+\sigma}$ and using (4.16) we get

$$
\forall t \geq 0, \quad \int_{t}^{+\infty} g(\tau)^{1+\sigma}(1+\tau)^{\sigma^{\prime}(1+\sigma)} d \tau \leq c g(t) .
$$

Define $\phi(t)=(1+t)^{\sigma^{\prime}(1+\sigma)+1}-1$ then $\phi(0)=0, \phi(t) \rightarrow+\infty$ as $t \rightarrow+\infty$ and $\phi^{\prime}(t)=\left(\sigma^{\prime}(1+\sigma)+1\right)(1+t)^{\sigma^{\prime}(1+\sigma)}$. Replacing $\phi^{\prime}$ on (4.18) we obtain

$$
\int_{t}^{\infty} g(\tau)^{1+\sigma} \phi^{\prime}(\tau) d \tau \leq \underbrace{c\left[\sigma^{\prime}(1+\sigma)+1\right]}_{=\frac{1}{w}} g(t)
$$

and since $g(0)=f(0)=1$, we can apply Lemma 4.1, to get

$$
g(t) \leq g(0)\left(\frac{1+\sigma}{1+w \sigma \phi(t)}\right)^{\frac{1}{\sigma}}=\left(\frac{1+\sigma}{1+w \sigma\left\{(1+t)^{\frac{1}{w c}}-1\right\}}\right)^{\frac{1}{\sigma}} .
$$

Define $r=\min \{1, w \sigma\}$ then $1+w \sigma\left\{(1+t)^{\frac{1}{w c}}-1\right\}>r\left\{1+(1+t)^{\frac{1}{w c}}-1\right\}=$ $r(1+t)^{\frac{1}{w c}}$, that is $\frac{1}{r(1+t)^{\frac{1}{w c}}}>\frac{1}{1+w \sigma\left\{(1+t)^{\frac{1}{w c}}-1\right\}}$, from where we can deduce that $g$ decays like

$$
g(t) \leq \frac{C}{(1+t)^{\frac{\left(\sigma^{\prime}(1+\sigma)+1\right)}{\sigma}}}=\frac{C}{(1+t)^{\sigma^{\prime}}(1+t)^{\frac{1+\sigma^{\prime}}{\sigma}}} .
$$

Thus, (4.17) holds.

Lemma 4.2. Let $f: \mathbb{R}^{+} \rightarrow \mathbb{R}^{+}$be a continuous and no increasing function. Let us assume that there are $\sigma>0, \sigma^{\prime}>0$ and $c>0$ such that

$$
\forall t>0, \quad \int_{t}^{\infty} f(\tau)^{1+\sigma} d \tau \leq c f(t)^{1+\sigma}+\frac{c f(0)^{\sigma} f(t)}{(1+t)^{\sigma^{\prime}}} .
$$


Then, there exists $C>0$ such that

$$
\forall t>0, \quad f(t) \leq f(0) \frac{C}{(1+t)^{\frac{\left(1+\sigma^{\prime}\right)}{\sigma}}} .
$$

Proof.- Is enough to prove the case $f(0)=1$, because if $1 \neq f(0)=d>0$, we define $g(t)=\frac{f(t)}{f(0)}$, then $g(0)=1$ and

$$
\begin{aligned}
\int_{t}^{\infty} g(\tau)^{1+\sigma} d \tau & =\frac{1}{f(0)^{1+\sigma}} \int_{t}^{+\infty} f(\tau)^{1+\sigma} d \tau \\
& \leq \frac{1}{f(0)^{1+\sigma}}\left\{c f(t)^{1+\sigma}+\frac{c f(0)^{\sigma} f(t)}{(1+t)^{\sigma^{\prime}}}\right\} \\
& =c g(t)^{1+\sigma}+c \frac{g(t)}{(1+t)^{\sigma^{\prime}}} .
\end{aligned}
$$

Thus, (4.19) holds for $g$ with $g(0)=1$. Then (4.20) holds for $g$ :

$$
g(t) \leq g(0) \frac{C}{(1+t)^{\frac{\left(1+\sigma^{\prime}\right)}{\sigma}}},
$$

Hence we obtain

$$
f(t) \leq f(0) \frac{C}{(1+t)^{\frac{\left(1+\sigma^{\prime}\right)}{\sigma}}} .
$$

Now, we prove for $f(0)=1$.

We will prove (4.20) by induction. Next, we denote por $C$ every constant. First, let us bound the right hand of (4.19),

$$
\begin{aligned}
c f(t)^{1+\sigma}+\frac{c f(0)^{\sigma} f(t)}{(1+t)^{\sigma^{\prime}}} & \leq c f(t)\left\{f(t)^{\sigma}+\frac{f(0)^{\sigma}}{(1+t)^{\sigma^{\prime}}}\right\} \\
& \leq c f(t)\left\{f(0)^{\sigma}+\frac{f(0)^{\sigma}}{(1+t)^{\sigma^{\prime}}}\right\} \\
& =c f(0) f(0)^{\sigma}\{1+\underbrace{\frac{1}{(1+t)^{\sigma^{\prime}}}}_{<1}\} \\
& <2 c f(0)^{1+\sigma},
\end{aligned}
$$

and so we have

$$
\int_{t}^{\infty} f(\tau)^{1+\sigma} d \tau \leq C f(t)
$$


Now, we prove that $\int_{t}^{\infty} f(\tau)^{1+\sigma} d \tau \leq C f(t)$ imply $f(t) \leq \frac{C}{(1+t)^{\frac{1}{\sigma}}}$.

In fact, considering $f(t)$ instead of $E(t), \phi(t)=t$ (i.e. $\phi^{\prime}(t)=1$, $\phi(0)=0, \phi(t) \longrightarrow+\infty$ as $t \rightarrow+\infty), \sigma>0$, and using (4.21) we deduce from Lemma 4.1 that

$$
f(t) \leq\left(\frac{1+\sigma}{1+\frac{\sigma}{C} t}\right)^{\frac{1}{\sigma}} .
$$

Taking $r:=\min \left\{1, \frac{\sigma}{C}\right\}$ then $r(1+t) \leq 1+\frac{\sigma}{C} t$, that is $\frac{1}{1+\frac{\sigma}{C} t} \leq \frac{1}{r(1+t)}$, from where we get

$$
f(t) \leq \frac{C}{(1+t)^{\frac{1}{\sigma}}}
$$

That is $f(t)^{\sigma} \leq \frac{C^{\sigma}}{1+t}$. Then, using this estimate in (4.19) we obtain

$$
\int_{t}^{+\infty} f(\tau)^{1+\sigma} d \tau \leq C \frac{f(t)}{1+t}+C \frac{f(t)}{(1+t)^{\sigma^{\prime}}} .
$$

Taking $\sigma_{1}:=\inf \left\{1, \sigma^{\prime}\right\}$, we have

$$
\int_{t}^{+\infty} f(\tau)^{1+\sigma} d \tau \leq C \frac{f(t)}{(1+t)^{\sigma_{1}}}
$$

and using Corollary 4.1 we arrive at

$$
f(t) \leq \frac{C}{(1+t)^{\frac{\left(1+\sigma_{1}\right)}{\sigma}}} .
$$

If $\sigma^{\prime} \leq 1$ then $\sigma_{1}=\sigma^{\prime}$, from where we get the inequality (4.20). If $\sigma^{\prime}>1$ then

$$
f(t) \leq \frac{C}{(1+t)^{\frac{2}{\sigma}}},
$$

that is $f(t)^{\sigma} \leq \frac{C^{\sigma}}{(1+t)^{2}}$. Then, using this estimate on (4.19) we have

$$
\int_{t}^{+\infty} f(\tau)^{1+\sigma} d \tau \leq C \frac{f(t)}{(1+t)^{2}}+C \frac{f(t)}{(1+t)^{\sigma^{\prime}}} .
$$

Taking $\sigma_{2}:=\inf \left\{2, \sigma^{\prime}\right\}$, we have

$$
\int_{t}^{+\infty} f(\tau)^{1+\sigma} d \tau \leq C \frac{f(t)}{(1+t)^{\sigma_{2}}}
$$


and using Corollary 4.1 we arrive at

$$
f(t) \leq \frac{C}{(1+t)^{\frac{\left(1+\sigma_{2}\right)}{\sigma}}} .
$$

If $\sigma^{\prime} \leq 2$ then $\sigma_{2}=\sigma^{\prime}$, from where we get the inequality (4.20). If $\sigma^{\prime}>2$ then

$$
f(t) \leq \frac{C}{(1+t)^{\frac{3}{\sigma}}},
$$

that is $f(t)^{\sigma} \leq \frac{C^{\sigma}}{(1+t)^{3}}$, and so on. Then, the conclusion holds by induction.

Lemma 4.3. Let $E: \mathbb{R}^{+} \rightarrow \mathbb{R}^{+}$be a no increasing function and $\phi:$ $\mathbb{R}^{+} \rightarrow \mathbb{R}^{+}$an strictly increasing $C^{1}$ function such that,

$$
\phi(0)=0 \quad \text { and } \quad \phi(t) \longrightarrow+\infty \text { as } t \rightarrow+\infty .
$$

Let us assume that there are $\sigma>0, \sigma^{\prime}>0$ and $c>0$ such that

$$
\int_{s}^{+\infty} E(t)^{1+\sigma} \phi^{\prime}(t) d t \leq c E(s)^{1+\sigma}+c \frac{E(s)}{(1+\phi(s))^{\sigma^{\prime}}} .
$$

Then, there exists $C>0$ depending continuously on $E(0)$, satisfying

$$
\forall t>0, \quad E(t) \leq \frac{C}{(1+\phi(t))^{\frac{\left(1+\sigma^{\prime}\right)}{\sigma}}} .
$$

Proof.- Is enough to define $f(\tau)=E\left(\phi^{-1}(\tau)\right)$ and use Lemma 4.2.

In analogy to this Lemma, we have the next version.

Lemma 4.4. Let $E: \mathbb{R}^{+} \rightarrow \mathbb{R}^{+}$be a no increasing function and $\phi:$ $\mathbb{R}^{+} \rightarrow \mathbb{R}^{+}$an strictly increasing $C^{1}$ function such that

$$
\phi(t) \longrightarrow+\infty \text { as } t \rightarrow+\infty \text {. }
$$

Let us assume that there are $\sigma>0, \sigma^{\prime}>0$ and $c>0$ such that

$$
\forall s \geq 1, \quad \int_{s}^{+\infty} E(t)^{1+\sigma} \phi^{\prime}(t) d t \leq c E(s)^{1+\sigma}+c \frac{E(s)}{\phi(s)^{\sigma^{\prime}}} .
$$

Then there exists $C>0$ depending continuously on $E(1)$ satisfying

$$
\forall t \geq 1, \quad E(t) \leq \frac{C}{\phi(t)^{\frac{\left(1+\sigma^{\prime}\right)}{\sigma}}} .
$$




\section{Using the multiplier method}

Let $\left(u^{0}, u^{1}\right) \in H^{m+1}(\Omega) \times H^{m}(\Omega)$ satisfying the $m$ th - order compatibility condition. Then, the regularity given by (2.3) justifies the calculus we are going to do.

We know that problem (1.1)-(1.3) is dissipative.

\section{Lemma 5.1.}

$$
E^{\prime}(t)=-\int_{\Omega} a(x)\left|u_{t}\right|^{2} d x, \quad \forall t>0 .
$$

Proof.- Multiplying the equation (1.1) by $u_{t}$ and integrating on $\Omega$, and using the Green Identity, we have

$$
\begin{aligned}
0 & =\int_{\Omega}\left(u_{t t}-\Delta u+a(x) u_{t}\right) u_{t} d x \\
& =\frac{\partial}{\partial t}\left\{\frac{1}{2} \int_{\Omega}\left(u_{t}\right)^{2} d x\right\}-\int_{\Omega}(\Delta u) u_{t} d x+\int_{\Omega} a(x)\left(u_{t}\right)^{2} d x \\
& =\frac{\partial}{\partial t}\left\{\frac{1}{2} \int_{\Omega}\left(u_{t}\right)^{2} d x\right\}+\int_{\Omega}(\nabla u) \nabla u_{t} d x+\int_{\Omega} a(x)\left(u_{t}\right)^{2} d x \\
& =\frac{\partial}{\partial t}\left\{\frac{1}{2} \int_{\Omega}\left(u_{t}\right)^{2} d x\right\}+\frac{\partial}{\partial t}\left\{\frac{1}{2} \int_{\Omega}|\nabla u|^{2} d x\right\}+\int_{\Omega} a(x)\left(u_{t}\right)^{2}, d x
\end{aligned}
$$

then the result holds.

Let $\sigma \geq 0$, and $\phi: \mathbf{R}^{+} \rightarrow \mathbf{R}^{+}$be a concave and increasing $C^{2}$ function. Let $w$ be a neighborhood of the boundary $\partial \Omega$.

Lemma 5.2. Let $h: \bar{\Omega} \longrightarrow \mathbb{R}^{N}$ be a $C^{1}$ vector field, $\sigma \geq 0$ and $0 \leq S \leq$ $T<+\infty$. Then we have,

$$
\begin{aligned}
& \int_{S}^{T} E^{\sigma} \phi^{\prime} \int_{\partial \Omega} 2 \partial_{\nu} u h . \nabla u+(h \cdot \nu)\left(\left|u_{t}\right|^{2}-|\nabla u|^{2}\right) \\
& =\left[E^{\sigma} \phi^{\prime} \int_{\Omega} q u_{t} h \cdot \nabla u\right]_{S}^{T}-\int_{S}^{T}\left(\sigma E^{\prime} E^{\sigma-1} \phi^{\prime}+E^{\sigma} \phi^{\prime \prime}\right) \int_{\Omega} 2 u_{t} h \cdot \nabla u \\
& +\int_{S}^{T} E^{\sigma} \phi^{\prime} \int_{\Omega}(\operatorname{div} h)\left(\left|u_{t}\right|^{2}-|\nabla u|^{2}\right)+2 \sum_{i, j} \frac{\partial h_{k}}{\partial x_{i}} \frac{\partial u}{\partial x_{i}} \frac{\partial u}{\partial x_{k}}+2 a u_{t} h \cdot \nabla u .
\end{aligned}
$$

Proof.- Multiplying the equation (1.1) by $E^{\sigma} \phi^{\prime} 2 h \cdot \nabla u$ and integrating on $[S, T] \times \Omega$ we have

$$
0=\int_{S}^{T} E^{\sigma} \phi^{\prime} \int_{\Omega} 2 h \cdot \nabla u\left(u_{t t}-\Delta u+a(x) u_{t}\right) d x d t
$$




$$
\begin{aligned}
= & \underbrace{\int_{S}^{T} E^{\sigma} \phi^{\prime} \int_{\Omega} 2 h \cdot(\nabla u) u_{t t} d x d t}_{I_{1}:=} \underbrace{-\int_{S}^{T} E^{\sigma} \phi^{\prime} \int_{\Omega} 2 h \cdot \nabla u(\Delta u) d x d t}_{I_{2}:=}+ \\
& \int_{S}^{T} E^{\sigma} \phi^{\prime} \int_{\Omega} 2 h \cdot \nabla u\left(a(x) u_{t}\right) d x . d t
\end{aligned}
$$

Since $\frac{\partial}{\partial t}\left(\int_{\Omega} 2 h(\nabla u) u_{t} d x\right)=\int_{\Omega} 2 h\left(\nabla u_{t}\right) u_{t} d x+\int_{\Omega} 2 h(\nabla u) u_{t t} d x$ and integrating by parts we obtain

$$
\begin{aligned}
I_{1}= & \int_{S}^{T} E^{\sigma} \phi^{\prime} \frac{\partial}{\partial t}\left(\int_{\Omega} 2 h(\nabla u) u_{t} d x\right) d t-\int_{S}^{T} E^{\sigma} \phi^{\prime} \int_{\Omega} 2 h\left(\nabla u_{t}\right) u_{t} d x d t \\
= & -\int_{S}^{T} E^{\sigma} \phi^{\prime} \int_{\Omega} 2 h(\nabla u) u_{t} d x d t+\left[E^{\sigma} \phi^{\prime} \int_{\Omega} 2 h(\nabla u) u_{t} d x\right]_{S}^{T} \\
& -\int_{S}^{T} E^{\sigma} \phi^{\prime} \int_{\Omega} 2 h\left(\nabla u_{t}\right) u_{t} d x d t .
\end{aligned}
$$

Using the Green Identity we have

$$
\begin{aligned}
I_{2} & =-\int_{S}^{T} E^{\sigma \phi^{\prime}} \int_{\Omega} 2 h \cdot \nabla u \Delta u d x d t \\
& =\int_{S}^{T} E^{\sigma} \phi^{\prime} \int_{\Omega} \nabla u \cdot \nabla(2 h \cdot \nabla u) d x d t-\int_{S}^{T} E^{\sigma} \phi^{\prime} \int_{\partial \Omega} \frac{\partial u}{\partial \nu}(2 h \cdot \nabla u) d t .
\end{aligned}
$$

Replacing $I_{1}$ and $I_{2}$ on the equality (5.3) we obtain

$$
\begin{aligned}
0= & -\int_{S}^{T} E^{\sigma} \phi^{\prime} \int_{\Omega} 2 h(\nabla u) u_{t} d x d t+\left[E^{\sigma} \phi^{\prime} \int_{\Omega} 2 h(\nabla u) u_{t} d x\right]_{S}^{T} \\
& \underbrace{-\int_{S}^{T} E^{\sigma} \phi^{\prime} \int_{\Omega} 2 h\left(\nabla u_{t}\right) u_{t} d x d t}_{I_{3}:=}+\underbrace{\int_{S}^{T} E^{\sigma} \phi^{\prime} \int_{\Omega} \nabla u \cdot \nabla(2 h \cdot \nabla u) d x d t}_{I_{4}:=} \\
& -\int_{S}^{T} E^{\sigma} \phi^{\prime} \int_{\partial \Omega} \frac{\partial u}{\partial \nu}(2 h \cdot \nabla u) d t+\int_{S}^{T} E^{\sigma} \phi^{\prime} \int_{\Omega} 2 h \cdot \nabla u\left(a(x) u_{t}\right) d x \cdot d t
\end{aligned}
$$

Using the fact that $h \nabla\left(u_{t}^{2}\right)=\operatorname{div}\left(h u_{t}^{2}\right)-(\operatorname{div} h) u_{t}^{2}$ and the Divergence Theorem $\int_{\partial \Omega} h u_{t}^{2} \cdot \nu=\int_{\Omega} \operatorname{div}\left(h u_{t}^{2}\right) d x$, we have

$$
\begin{aligned}
I_{3} & =-\int_{S}^{T} E^{\sigma} \phi^{\prime} \int_{\Omega} h \cdot \nabla\left(u_{t}^{2}\right) d x d t \\
& =-\int_{S}^{T} E^{\sigma} \phi^{\prime} \int_{\Omega} \operatorname{div}\left(h u_{t}^{2}\right) d x d t+\int_{S}^{T} E^{\sigma} \phi^{\prime} \int_{\Omega}(\operatorname{div} h) u_{t}^{2} d x d t \\
& =-\int_{S}^{T} E^{\sigma} \phi^{\prime} \int_{\partial \Omega}\left(h u_{t}^{2}\right) \cdot \nu d x d t+\int_{S}^{T} E^{\sigma} \phi^{\prime} \int_{\Omega}(\operatorname{div} h) u_{t}^{2} d x d t .
\end{aligned}
$$


By other hand, we have

$$
\nabla u \cdot \nabla(2 h \cdot \nabla u)=2 \partial_{i} u \partial_{i} h_{k} \partial_{k} u+h \cdot \nabla\left(|\nabla u|^{2}\right) .
$$

And since $\operatorname{div}\left(h|\nabla u|^{2}\right)=(\operatorname{divh})|\nabla u|^{2}+h \nabla\left(|\nabla u|^{2}\right)$ and using the Divergence Theorem, we have

$$
\begin{aligned}
\int_{\Omega}(\operatorname{div} h)|\nabla u|^{2} d x+\int_{\Omega} h \nabla\left(|\nabla u|^{2}\right) d x & =\int_{\Omega} \operatorname{div}\left(h|\nabla u|^{2}\right) d x \\
& =\int_{\partial \Omega} h|\nabla u|^{2} \cdot \nu d x
\end{aligned}
$$

Using (5.5) and (5.6) we obtain

$$
\begin{aligned}
I_{4}= & 2 \int_{S}^{T} E^{\sigma} \phi^{\prime} \int_{\Omega} \partial_{i} u \partial_{i} h_{k} \partial_{k} u d x d t+\int_{S}^{T} E^{\sigma} \phi^{\prime} \int_{\Omega} h \cdot \nabla\left(|\nabla u|^{2}\right) d x d t \\
= & 2 \int_{S}^{T} E^{\sigma} \phi^{\prime} \int_{\Omega} \partial_{i} u \partial_{i} h_{k} \partial_{k} u d x d t+\int_{S}^{T} E^{\sigma} \phi^{\prime} \int_{\partial \Omega} h|\nabla u|^{2} \cdot \nu d x d t \\
& -\int_{S}^{T} E^{\sigma} \phi^{\prime} \int_{\Omega}(\operatorname{div} h)|\nabla u|^{2} d x d t .
\end{aligned}
$$

Replacing $I_{3}$ and $I_{4}$ in the equality (5.4), we will have the result.

Lemma 5.3. There exists a constant $C>0$ such that $\forall 0 \leq S<T<\infty$

(5.7) $\int_{S}^{T} E(t)^{1+\sigma} \phi^{\prime}(t) d t \leq C E(S)^{1+\sigma}+C \int_{S}^{T} E(t)^{\sigma} \phi^{\prime}(t)\left(\int_{w}\left|u_{t}\right|^{2} d x\right) d t$.

holds.

Proof.- Let $K_{1}$ be a compact of $\Omega$ such that $\Omega-K_{1}$ be a compact set on $w$.

Define $h(x):=\beta(x) m(x)$, where $m(x)=x-x_{0}$ and $\beta$ is a $C^{\infty}$ function whoose support is compactly in $\Omega$ and equal to 1 on $K_{1}$. Since $\phi^{\prime}$ is no increasing and positive then $\phi^{\prime}$ is bounded on $\mathbb{R}^{+}$(i.e. $\left|\phi^{\prime}(t)\right| \leq M$ ). 
Now, we apply (5.2) to this $h$ and get

$$
\begin{aligned}
& 0 \geq\left[E^{\sigma} \phi^{\prime} \int_{\Omega} 2 u_{t} h \cdot \nabla u d x\right]_{S}^{T}-\int_{S}^{T}\left(\sigma E^{\prime} E^{\sigma-1} \phi^{\prime}+E^{\sigma} \phi^{\prime \prime}\right)\left(\int_{\Omega} 2 u_{t} h \cdot \nabla u d x\right) d t \\
& +\int_{S}^{T} E^{\sigma} \phi^{\prime} \int_{\Omega} \operatorname{div} h\left(u_{t}^{2}-|\nabla u|^{2}\right)+2 \sum_{i, j} \frac{\partial h_{k}}{\partial x_{i}} \frac{\partial u}{\partial x_{i}} \frac{\partial u}{\partial x_{k}}+2 a(x) u_{t} h \cdot \nabla u .
\end{aligned}
$$

By other hand, using that $\int_{\Omega} 2 h \cdot \nabla u u_{t} d x \leq c E(t)$ and $E(t)^{\sigma+1}<$ $E(S)^{\sigma+1}$ para $S<T$, we have

$$
\begin{aligned}
& \left|\int_{S}^{T}\left(\sigma E^{\prime} E^{\sigma-1} \phi^{\prime}+E^{\sigma} \phi^{\prime \prime}\right)\left(\int_{\Omega} 2 h \cdot \nabla u u_{t} d x\right) d t\right| \\
& \quad \leq \int_{S}^{T}\left|\left(\sigma E^{\prime} E^{\sigma-1} \phi^{\prime}+E^{\sigma} \phi^{\prime \prime}\right)\left(\int_{\Omega} 2 h \cdot \nabla u u_{t} d x\right)\right| d t \\
& \quad \leq \int_{S}^{T}\left|\sigma E^{\prime} E^{\sigma-1} \phi^{\prime}+E^{\sigma} \phi^{\prime \prime}\right| c E d t \\
& \quad=\int_{S}^{T}\left\{-\sigma E^{\prime} E^{\sigma-1} \phi^{\prime}-E^{\sigma} \phi^{\prime \prime}\right\} c E d t \\
& \quad \leq c M \int_{S}^{T}-\sigma E^{\prime} E^{\sigma} d t+c E(S)^{\sigma+1} \int_{S}^{\int_{S}^{T}-\phi^{\prime \prime} d t} \\
& \quad=c M\left[\frac{\sigma}{\sigma+1} E(t)^{\sigma+1}\right]_{T}^{S}+c E(S)^{\sigma+1} \underbrace{\left[\phi^{\prime}\right]_{T}^{S}}_{\leq 2 M} \\
& \quad \leq c M \frac{\sigma}{\sigma+1} E(S)^{\sigma+1}+c 2 M E(S)^{\sigma+1} \\
& \quad \leq c^{\prime} E(S)^{\sigma+1} .
\end{aligned}
$$

And, since $E(T)<E(S)$ and by Holder $\int_{\Omega} u_{t} h \cdot \nabla u d x \leq\left\|u_{t}\right\|\|\nabla u\| \leq$ $E(t)$, we obtain

$$
\begin{aligned}
-\left[E^{\sigma} \phi^{\prime} \int_{\Omega} 2 u_{t} h \cdot \nabla u d x\right]_{S}^{T} \leq & -E^{\sigma}(T) \phi^{\prime}(T) \int_{\Omega} 2 u_{t}(T) h(T) \cdot \nabla u(T) d x \\
& +E^{\sigma}(S) \phi^{\prime}(S) \int_{\Omega} 2 u_{t}(S) h(S) \cdot \nabla u(S) d x \\
\leq & E^{\sigma}(S) C\{E(S)+E(T)\} \leq C E^{\sigma+1}(S) .
\end{aligned}
$$


Here we need to make the following estimate

$$
\begin{gathered}
-\int_{S}^{T} E^{\sigma} \phi^{\prime} \int_{\Omega} 2 \sum_{i, j} \frac{\partial h_{k}}{\partial x_{i}} \frac{\partial u}{\partial x_{i}} \frac{\partial u}{\partial x_{k}} d x d t \leq \int_{S}^{T} E^{\sigma} \phi^{\prime} c\|\nabla u\|\|\nabla u\|_{L^{2}\left(\Omega-K_{1}\right)} d t \\
\leq \int_{S}^{T} E^{\sigma} \phi^{\prime}\left\{\epsilon E(t)+C(\epsilon)\|\nabla u\|_{L^{2}\left(\Omega-K_{1}\right)}^{2}\right\} d t
\end{gathered}
$$

where $\epsilon$ will be considered little enough.

Using the inequalities (5.9), (5.10) and (5.11) on (5.8) we have that there exists $C>0$ such that

$$
\begin{gathered}
\int_{S}^{T} E^{\sigma} \phi^{\prime} \int_{\Omega} N u_{t}^{2}+(2-N)|\nabla u|^{2} d x d t \\
\leq C \int_{S}^{T} E^{\sigma} \phi^{\prime} \int_{\Omega-K_{1}}\left\{u_{t}^{2}+|\nabla u|^{2}\right\} d x d t+C E(S)^{1+\sigma}+\epsilon \int_{S}^{T} E^{1+\sigma} \phi^{\prime} d t
\end{gathered}
$$

with $\epsilon$ little enough.

Integrating by parts the expression:

$$
0=(N-1) \int_{S}^{T} E^{\sigma} \phi^{\prime} \int_{\Omega} u\left(u_{t t}-\Delta u+a(x) u_{t}\right) d x d t
$$

we have

$$
\begin{aligned}
(N-1) \int_{S}^{T} E^{\sigma} \phi^{\prime} \int_{\Omega}|\nabla u|^{2} d x d t-(N-1) \int_{S}^{T} E^{\sigma} \phi^{\prime} \int_{\Omega}\left|u_{t}\right|^{2} d x d t \\
=-(N-1)\left[E^{\sigma} \phi^{\prime} \int_{\Omega} u u_{t} d x\right]_{S}^{T}-(N-1) \int_{S}^{T} E^{\sigma} \phi^{\prime} \int_{\Omega} u a u_{t} d x d t \\
\quad+(N-1) \int_{S}^{T}\left(\sigma E^{\sigma-1} E^{\prime} \phi^{\prime}+E^{\sigma} \phi^{\prime \prime}\right) \int_{\Omega} u u_{t} d x d t
\end{aligned}
$$


By the Poincaré inequality we have $\int_{\Omega} u u_{t} d x \leq\|u\|\left\|u_{t}\right\| \leq C_{p}\|\nabla u\|\left\|u_{t}\right\| \leq$ $C E(t)$. Using this in (5.9) we obtain

$$
\left|(N-1) \int_{S}^{T}\left(\sigma E^{\sigma-1} E^{\prime} \phi^{\prime}+E^{\sigma} \phi^{\prime \prime}\right) \int_{\Omega} u u_{t} d x d t\right| \leq C E(S)^{\sigma+1}
$$

With a similar proof to (5.10), we also obtain

$$
-(N-1)\left[E^{\sigma} \phi^{\prime} \int_{\Omega} u u_{t} d x\right]_{S}^{T} \leq C E(S)^{\sigma+1} .
$$

Adding (5.12) and (5.13), taking $\epsilon<1$ and using (5.14) and (5.15) we have

$$
\begin{aligned}
\int_{S}^{T} E^{1+\sigma} \phi^{\prime} d t & \leq \int_{S}^{T} E^{\sigma} \phi^{\prime} \int_{\Omega} u_{t}^{2}+|\nabla u|^{2} d x d t \\
& \leq C E(S)^{1+\sigma}+C \int_{S}^{T} E^{\sigma} \phi^{\prime} \int_{\Omega-K_{1}}\left\{u_{t}^{2}+|\nabla u|^{2}\right\} d x d t
\end{aligned}
$$

We want to eliminate the last term of (5.16). To do this, we construct a function $\xi \in C^{\infty}(\bar{\Omega})$ such that $\xi=1$ in $\Omega-K_{1}$ and $\xi=0$ outside $w$.

We multiply the equation (1.1) by $\xi u$ and integrate it on $\Omega$; then we multiply this expression by $E^{\sigma} \phi^{\prime}$, and integrate on $[S, T]$, and integrating by parts we get

$$
\begin{aligned}
& \int_{S}^{T} E^{\sigma} \phi^{\prime} \int_{\Omega}-\xi u a(x) u_{t} d x d t \\
& =\int_{S}^{T} E^{\sigma} \phi^{\prime} \int_{\Omega} \xi u\left(u_{t t}-\Delta u\right) d x d t \\
& =\int_{S}^{T} E^{\sigma} \phi^{\prime} \int_{\Omega} \xi u u_{t t} d x d t-\int_{S}^{T} E^{\sigma} \phi^{\prime} \int_{\Omega} \xi u \Delta u d x d t \\
& =-\int_{S}^{T}\left(\sigma E^{\sigma-1} E^{\prime} \phi^{\prime}+E^{\sigma} \phi^{\prime \prime}\right) \int_{\Omega} \xi u u_{t} d x d t \\
& -\int_{S}^{T} E^{\sigma} \phi^{\prime} \int_{\Omega} \xi u_{t}^{2} d x d t+\left[E^{\sigma} \phi^{\prime} \int_{\Omega} u u_{t} d x\right]_{S}^{T} \\
& \underbrace{-\int_{S}^{T} E^{\sigma} \phi^{\prime} \int_{\Omega} \xi u \Delta u d x d t}_{I:=} .
\end{aligned}
$$


Using the Green Identity and $\nabla\left(u^{2}\right)=(\nabla u) u+u \nabla u$ we have

$$
\begin{aligned}
I & =\int_{S}^{T} E^{\sigma} \phi^{\prime} \int_{\Omega} \nabla(\xi u) \cdot \nabla u d x d t \\
& =\int_{S}^{T} E^{\sigma} \phi^{\prime} \int_{\Omega}\{\nabla(\xi) u \cdot \nabla u+\xi \nabla u \cdot \nabla u\} d x d t \\
& =\int_{S}^{T} E^{\sigma} \phi^{\prime} \int_{\Omega}\left\{\frac{1}{2} \nabla(\xi) \cdot \nabla\left(u^{2}\right)+\xi|\nabla u|^{2}\right\} d x d t \\
& =\int_{S}^{T} E^{\sigma} \phi^{\prime} \int_{\Omega}\left\{-\frac{1}{2}(\Delta \xi) u^{2}+\xi|\nabla u|^{2}\right\} d x . d t
\end{aligned}
$$

Replacing (5.18) on (5.17) we obtain

$$
\begin{aligned}
\int_{S}^{T} E^{\sigma} \phi^{\prime} \int_{\Omega}-\xi u a(x) u_{t} d x d t= & -\int_{S}^{T}\left(\sigma E^{\sigma-1} E^{\prime} \phi^{\prime}+E^{\sigma} \phi^{\prime \prime}\right) \int_{\Omega} \xi u u_{t} d x d t \\
& +\int_{S}^{T} E^{\sigma} \phi^{\prime} \int_{\Omega} \xi|\nabla u|^{2} d x d t+\left[E^{\sigma} \phi^{\prime} \int_{\Omega} u u_{t} d x\right]_{S}^{T} \\
& -\int_{S}^{T} E^{\sigma} \phi^{\prime} \int_{\Omega}\left\{\frac{1}{2}(\Delta \xi) u^{2}+\xi u_{t}^{2}\right\} d x d t
\end{aligned}
$$

from where

$$
\begin{aligned}
\int_{S}^{T} E^{\sigma} \phi^{\prime} \int_{\Omega-K_{1}} 1 \cdot|\nabla u|^{2} d x d t \leq & \int_{S}^{T} E^{\sigma} \phi^{\prime} \int_{\Omega} \xi|\nabla u|^{2} d x d t \\
= & \int_{S}^{T}\left(\sigma E^{\sigma-1} E^{\prime} \phi^{\prime}+E^{\sigma} \phi^{\prime \prime}\right) \int_{\Omega} \xi u u_{t} d x d t \\
& -\left[E^{\sigma} \phi^{\prime} \int_{\Omega} u u_{t} d x\right]_{S}^{T} \\
& +\int_{S}^{T} E^{\sigma} \phi^{\prime} \int_{\Omega}-\xi u a(x) u_{t} d x d t \\
& +\int_{S}^{T} E^{\sigma} \phi^{\prime} \int_{\Omega}\left\{\frac{1}{2}(\Delta \xi) u^{2}+\xi u_{t}^{2}\right\} d x d t .
\end{aligned}
$$

Since $\xi$ is bounded, in a similar way to (5.9), we obtain

$$
\left|\int_{S}^{T}\left(\sigma E^{\sigma-1} E^{\prime} \phi^{\prime}+E^{\sigma} \phi^{\prime \prime}\right) \int_{\Omega} \xi u u_{t} d x d t\right| \leq C E^{1+\sigma} .
$$

Also, using the fact of $\xi$ is bounded, similarly to (5.10) we get

$$
-\left[E^{\sigma} \phi^{\prime} \int_{\Omega} u u_{t} d x\right]_{S}^{T} \leq C E^{1+\sigma} .
$$


And, using (5.21) and (5.22) in (5.20) we obtain

$$
\int_{S}^{T} E^{\sigma} \phi^{\prime} \int_{\Omega-K_{1}}|\nabla u|^{2} d x d t \leq C E(S)^{1+\sigma}+C \int_{S}^{T} E^{\sigma} \phi^{\prime} \int_{w}\left(u_{t}^{2}+u^{2}\right) d x d t .
$$

Now, to eliminate the last term of (5), we will adapt the Conrad and Rao [1] method.

We start with an arbitrary function $\beta \in C^{\infty}\left(\mathbf{R}^{N}\right)$ be such that $0 \leq \beta \leq$ $1, \beta=1$ on $w$ and $\beta=0$ outside a neighborhood of $w$ (see [4], Theorem 1.2 .2 , or [2] p.p 3489).

Now, fix $t$ and consider the solution $z$ to the elliptic problem:

$$
\begin{aligned}
\Delta z & =\beta(x) u \text { in } \Omega \\
\left.z\right|_{\partial \Omega} & =0 .
\end{aligned}
$$

Multiplying the equation (5.24) by $z$, integrating on $\Omega$ and using the Green Identity, we have:

$$
\int_{\Omega} \beta(x) u z d x=\int_{\Omega}(\Delta z) z d x=-\int_{\Omega}|\nabla z|^{2} d x,
$$

hence, using the Holder and Poincaré inequalities, we obtain

$$
|z|_{L^{2}}^{2} \leq C \int_{\Omega}|\nabla z|^{2} d x=-C \int_{\Omega} \beta(x) u z d x \leq c|u|_{L^{2}(\Omega)}|z|_{L^{2}(\Omega)}
$$

then,

$$
|z|_{L^{2}} \leq C|u|_{L^{2}(\Omega)} .
$$

Similarly to $(5.26)$ we have

$$
\begin{aligned}
|z|_{L^{2}}^{2} \leq C \int_{\Omega}|\nabla z|^{2} d x=-C \int_{\Omega} \beta(x) u z d x & \leq c|\beta u|_{L^{2}(\Omega)}|z|_{L^{2}(\Omega)} \\
& \leq c|u|_{L^{2}(w)}|z|_{L^{2}(\Omega)},
\end{aligned}
$$

then,

$$
|z|_{L^{2}} \leq C|u|_{L^{2}(w)}
$$


Differentiating with respect to $t$ to the equation (5.24) we have the problem

$$
\begin{aligned}
\Delta z_{t} & =\beta(x) u_{t} \text { in } \Omega \\
\left.z_{t}\right|_{\partial \Omega} & =0 .
\end{aligned}
$$

Multiplying (5.30) by $z_{t}$, integrating on $\Omega$ and using the Green Identity we obtain

$$
\int_{\Omega} \beta(x) u_{t} z_{t} d x=\int_{\Omega}\left(\Delta z_{t}\right) z_{t} d x=-\int_{\Omega}\left|\nabla z_{t}\right|^{2} d x,
$$

hence, using the Holder and Poincaré inequalities, we obtain

$(5.32)\left|z_{t}\right|_{L^{2}}^{2} \leq C \int_{\Omega}\left|\nabla z_{t}\right|^{2} d x=-C \int_{\Omega} \beta(x) u_{t} z_{t} d x \leq c\left|u_{t}\right|_{L^{2}(\Omega)}\left|z_{t}\right|_{L^{2}(\Omega)}$

then

$$
\left|z_{t}\right|_{L^{2}} \leq C\left|u_{t}\right|_{L^{2}(\Omega)}
$$

Also

$$
\begin{aligned}
\left|z_{t}\right|_{L^{2}}^{2} \leq C \int_{\Omega}\left|\nabla z_{t}\right|^{2} d x=-C \int_{\Omega} \beta(x) u_{t} z_{t} d x & \leq c\left|\beta u_{t}\right|_{L^{2}(\Omega)}\left|z_{t}\right|_{L^{2}(\Omega)} \\
& \leq c\left|u_{t}\right|_{L^{2}(w)}\left|z_{t}\right|_{L^{2}(\Omega)}
\end{aligned}
$$

then

$$
\left|z_{t}\right|_{L^{2}} \leq C\left|u_{t}\right|_{L^{2}(w)}
$$

By other hand,

$$
\begin{aligned}
0= & \int_{S}^{T} E^{\sigma} \phi^{\prime} \int_{\Omega} z\left(u_{t t}-\Delta u+a u_{t}\right) d x d t \\
= & {\left[E^{\sigma} \phi^{\prime} \int_{\Omega} z u_{t} d x\right]_{S}^{T}-\int_{S}^{T}\left(\sigma E^{\prime} E^{\sigma-1} \phi^{\prime}+E^{\sigma} \phi^{\prime \prime}\right) \int_{\Omega} z u_{t} d x d t } \\
& +\int_{S}^{T} E^{\sigma} \phi^{\prime} \int_{\Omega}\left(-z \Delta u+a z u_{t}-z_{t} u_{t}\right) d x d t .
\end{aligned}
$$

Using the Green Identity and the fact that $z$ is solution of (5.24)-(5.25) we obtain

$$
\begin{aligned}
\int_{S}^{T} E^{\sigma} \phi^{\prime} \int_{\Omega} z \Delta u d x d t & =\int_{S}^{T} E^{\sigma} \phi^{\prime} \int_{\Omega}(\Delta z) u d x d t \\
& =\int_{S}^{T} E^{\sigma} \phi^{\prime} \int_{\Omega}(\beta(x) u) u d x d t \\
& =\int_{S}^{T} E^{\sigma} \phi^{\prime} \int_{w} u^{2} d x d t
\end{aligned}
$$


Using (5.37) on (5.36) we have

$$
\begin{aligned}
\int_{S}^{T} E^{\sigma} \phi^{\prime} \int_{w} u^{2} d x d t= & \int_{S}^{T} E^{\sigma} \phi^{\prime} \int_{\Omega} z \Delta u d x d t \\
= & {\left[E^{\sigma} \phi^{\prime} \int_{\Omega} z u_{t} d x\right]_{S}^{T}-\int_{S}^{T}\left(\sigma E^{\prime} E^{\sigma-1} \phi^{\prime}+E^{\sigma} \phi^{\prime \prime}\right) \int_{\Omega} z u_{t} d x d t } \\
& +\int_{S}^{T} E^{\sigma} \phi^{\prime} \int_{\Omega}\left(a z u_{t}-z_{t} u_{t}\right) d x d t .
\end{aligned}
$$

We can observe that the following inequality holds

$$
\left|\left[E^{\sigma} \phi^{\prime} \int_{\Omega} z u_{t} d x\right]_{S}^{T}-\int_{S}^{T}\left(\sigma E^{\prime} E^{\sigma-1} \phi^{\prime}+E^{\sigma} \phi^{\prime \prime}\right) \int_{\Omega} z u_{t} d x d t\right| \leq C E(S)^{1+\sigma} .
$$

By other hand, let $\eta>0$, using (5.35) we obtain

$$
\begin{aligned}
\left|\int_{S}^{T} E^{\sigma} \phi^{\prime} \int_{\Omega} z_{t} u_{t} d x d t\right| & \leq \int_{S}^{T} E^{\sigma} \phi^{\prime} C\left|z_{t}\right|_{L^{2}}\left|u_{t}\right|_{L^{2}} d t \\
& \leq \int_{S}^{T} E^{\sigma} \phi^{\prime} C\left|u_{t}\right|_{L^{2}(w)}\left|u_{t}\right|_{L^{2}} d t \\
& \leq \frac{C}{2 \eta} \int_{S}^{T} E^{\sigma} \phi^{\prime} \int_{w} u_{t}^{2} d x d t+\frac{\eta}{2} \int_{S}^{T} E^{\sigma} \phi^{\prime} \int_{\Omega} u_{t}^{2} d x d t \\
& \leq \frac{C}{2 \eta} \int_{S}^{T} E^{\sigma} \phi^{\prime} \int_{w} u_{t}^{2} d x d t+\eta \int_{S}^{T} E^{\sigma+1} \phi^{\prime} d t
\end{aligned}
$$

where $\eta$ will be taken little enough.

Also, we have

$$
\begin{aligned}
\left|\int_{S}^{T} E^{\sigma} \phi^{\prime} \int_{\Omega} z a u_{t} d x d t\right| & \leq \int_{S}^{T} E^{\sigma} \phi^{\prime}\left|\int_{\Omega} z a u_{t} d x\right| d t \\
& \leq \int_{S}^{T} E^{\sigma} \phi^{\prime}|z|_{L^{2}(\Omega)}\left|a u_{t}\right|_{L^{2}} d t \\
& \leq \int_{S}^{T} E^{\sigma} \phi^{\prime} C|u|_{L^{2}(w)}\left|\sqrt{a} u_{t}\right|_{L^{2}} d t \\
& \leq \gamma \int_{S}^{T} E^{\sigma} \phi^{\prime} \int_{w} u^{2} d x d t+C(\gamma) \int_{S}^{T} E^{\sigma} \phi^{\prime} \int_{\Omega} a u_{t}^{2} d x d t \\
& =\gamma \int_{S}^{T} E^{\sigma} \phi^{\prime} \int_{w} u^{2} d x d t+C(\gamma) \int_{S}^{T} E^{\sigma} \phi^{\prime}\left(-E^{\prime}\right) d t \\
& \leq \gamma \int_{S}^{T} E^{\sigma} \phi^{\prime} \int_{w} u^{2} d x d t-C(\gamma) \int_{S}^{T} E^{\sigma}\left(E^{\prime}\right) d t,
\end{aligned}
$$


where $\gamma$ will be taken very little.

Since

$$
\begin{aligned}
-\int_{S}^{T} E^{\sigma} E^{\prime} d t=-\frac{1}{\sigma+1}\left[E(t)^{\sigma+1}\right]_{S}^{T} & =\frac{1}{\sigma+1}\left\{E(S)^{\sigma+1}-E(T)^{\sigma+1}\right\} \\
& \leq \frac{1}{\sigma+1}\left\{E(S)^{\sigma+1}\right\}
\end{aligned}
$$

then (5.41) becomes

$$
\left|\int_{S}^{T} E^{\sigma} \phi^{\prime} \int_{\Omega} z a u_{t} d x d t\right| \leq \gamma \int_{S}^{T} E^{\sigma} \phi^{\prime} \int_{w} u^{2} d x d t+C(\gamma) E(S)^{\sigma+1} .
$$

Using (5.39), (5.40) and (5.42) in (5.38), we have

$$
\begin{aligned}
(1-\gamma) \int_{S}^{T} E^{\sigma} \phi^{\prime} \int_{w} u^{2} d x d t \leq & \frac{C}{\eta} \int_{S}^{T} E^{\sigma} \phi^{\prime} \int_{w} u_{t}^{2} d x d t \\
& +C E(S)^{1+\sigma}+\eta \int_{S}^{T} E^{1+\sigma} \phi^{\prime} d t .
\end{aligned}
$$

Taking $\gamma<1$, from (5.43) we have that there exists $C>0$ such that $\forall \eta>0$

$$
\begin{aligned}
\int_{S}^{T} E^{\sigma} \phi^{\prime} \int_{w} u^{2} d x d t \leq & \frac{C}{\eta} \int_{S}^{T} E^{\sigma} \phi^{\prime} \int_{w} u_{t}^{2} d x d t \\
& +C E(S)^{1+\sigma}+\eta \int_{S}^{T} E^{1+\sigma} \phi^{\prime} d t
\end{aligned}
$$

holds, where $\eta$ is little enough.

Replacing (5.44) in (5.23) we get

$$
\begin{gathered}
\int_{S}^{T} E^{\sigma} \phi^{\prime} \int_{\Omega-K_{1}}|\nabla u|^{2} d x d t \\
(5.45) \leq C E(S)^{1+\sigma}+\left(C+\frac{C}{\eta}\right) \int_{S}^{T} E^{\sigma} \phi^{\prime} \int_{w} u_{t}^{2} d x d t+\eta \int_{S}^{T} E^{1+\sigma} \phi^{\prime} d t .
\end{gathered}
$$

Replacing (5.45) in (5.16) we have

$$
(1-\eta) \int_{S}^{T} E^{1+\sigma} \phi^{\prime} d t \leq C E(S)^{1+\sigma}+\left(C+\frac{C}{\eta}\right) \int_{S}^{T} E^{\sigma} \phi^{\prime} \int_{w} u_{t}^{2} d x d t
$$


and taking $\eta<1$ we obtain

$$
\int_{S}^{T} E^{1+\sigma} \phi^{\prime} d t \leq C E(S)^{1+\sigma}+C \int_{S}^{T} E^{\sigma} \phi^{\prime} \int_{w} u_{t}^{2} d x d t .
$$

\section{Finishing the proof of the Theorem}

Let $\rho: t \longrightarrow \rho(t)$ a decreasing function which goes to zero as $t$ goes to the infinite. Later on, we choose $\rho$.

Let us define the function $\tilde{\alpha}$ by

$$
\begin{array}{lll}
\tilde{\alpha}(r, t):=\tilde{a}(r) & \text { if } \quad & \frac{R}{2} \leq r \leq R-\rho(t) \\
\tilde{\alpha}(r, t):=\tilde{a}(R-\rho(t)) & \text { if } & r \geq R-\rho(t)
\end{array}
$$

and the function $\alpha$ on $w \times \mathbf{R}^{+}$by

$$
\alpha(x, t):=\tilde{\alpha}(|x|, t), \quad \forall|x| \geq \frac{R}{2} .
$$

Lemma 6.1 (Gagliardo-Niremberg). If $m>\frac{N}{2}$, there exists $c>0$ such that for every $v \in H^{m}(\Omega)$

$$
\|v\|_{L^{\infty}(\Omega)} \leq c\|v\|_{H^{m}(\Omega)}^{\theta}\|v\|_{L^{2}(\Omega)}^{1-\theta} \quad \text { with } \quad \theta=\frac{N}{2 m} .
$$

Now, using (2.3) and (2.4) it is possible to apply (6.3) and deduce

$$
\begin{aligned}
\left\|u_{t}\right\|_{L^{\infty}(\Omega)}^{2} & \leq c\left\|u_{t}\right\|_{H^{m}(\Omega)}^{2} \underbrace{\left\|u_{t}\right\|_{L^{2}(\Omega)}^{2(1-\theta)}}_{\leq E(t)^{1-\theta}} \\
& \leq C_{m}\left\|\left(u^{0}, u^{1}\right)\right\|_{H^{m+1} \times H^{m}}^{2 \theta} E(t)^{1-\theta}
\end{aligned}
$$

Let $p>0$ such that (2.6) holds. Then, using the Jensen Inequality and (6.4) we will estimate the last term of (5.7).

$$
\begin{aligned}
& \int_{S}^{T} E(t)^{\sigma} \phi^{\prime}(t)\left(\int_{w} u_{t}^{2} d x\right) d t \\
= & \int_{S}^{T} E(t)^{\sigma} \phi^{\prime}(t)\left(\int_{w} \frac{1}{\alpha(x, t)} u_{t}^{2} \alpha(x, t) d x\right) d t \\
\leq & \int_{S}^{T} E(t)^{\sigma} \phi^{\prime}(t)\left\|u_{t}^{2} \alpha^{\frac{p}{p+1}}\right\|_{L^{\frac{p+1}{p}}}\left\|\alpha^{-\frac{p}{p+1}}\right\|_{L^{p+1}} d t
\end{aligned}
$$




$$
\begin{aligned}
& =\int_{S}^{T} E(t)^{\sigma} \phi^{\prime}(t)\left(\int_{w} \frac{1}{\alpha(x, t)^{p}} d x\right)^{\frac{1}{p+1}}\left(\int_{w} u_{t}^{\frac{2(p+1)}{p}} \alpha(x, t) d x\right)^{\frac{p}{p+1}} d t \\
& =\quad \int_{S}^{T} E(t)^{\sigma} \phi^{\prime}(t)^{\frac{1}{(p+1)}}\left(\phi^{\prime}(t)^{p} \int_{w} \frac{1}{\alpha(x, t)^{p}} d x\right)^{\frac{1}{p+1}}\left(\int_{w} u_{t}^{2+\frac{2}{p}} \alpha(x, t) d x\right)^{\frac{p}{p+1}} d t \\
& \leq \quad \int_{S}^{T} E(t)^{\sigma} \phi^{\prime}(t)^{\frac{1}{(p+1)}}\left(\phi^{\prime}(t)^{p} \int_{w} \frac{1}{\alpha(x, t)^{p}} d x\right)^{\frac{1}{p+1}} \\
& \quad \cdot\left(\int_{w} u_{t}^{2} \alpha(x, t) d x\right)^{\frac{p}{p+1}}\left\|u_{t}(t)\right\|_{L^{\infty}(\Omega)}^{\frac{2}{p+1}} d t \\
& \leq \quad C_{m} \int_{S}^{T} E(t)^{\sigma+\frac{(1-\sigma)}{(p+1)}} \phi^{\prime}(t)^{\frac{1}{(p+1)}}\left(\phi^{\prime}(t)^{p} \int_{w} \frac{1}{\alpha(x, t)^{p}} d x\right)^{\frac{1}{p+1}} \\
& \quad \cdot\left(\int_{w} u_{t}^{2} \alpha(x, t) d x\right)^{\frac{p}{p+1}} d t .
\end{aligned}
$$

To simplify notations, we introduce

$$
\varepsilon(t)=\phi^{\prime}(t)\left(\int_{w} \frac{1}{\alpha(x, t)^{p}} d x\right)^{\frac{1}{p}} .
$$

Let $\epsilon>0$. Applying the Young inequality we get the following estimation

$$
\begin{aligned}
& \int_{S}^{T} E(t)^{\sigma} \phi^{\prime}(t)\left(\int_{w} \frac{1}{\alpha(x, t)^{p}} d x\right) d t \\
\leq & C_{m} \int_{S}^{T} E(t)^{\sigma+\frac{(1-\theta)}{(p+1)}} \phi^{\prime}(t)^{\frac{1}{(p+1)}} \varepsilon(t)^{\frac{p}{p+1}}\left(\int_{w} u_{t}^{2} \alpha(x, t) d x\right)^{\frac{p}{p+1}} d t \\
\leq & C_{m} \int_{S}^{T} \underbrace{E(t)^{\sigma+\frac{(1-\theta)}{(p+1)}} \phi^{\prime}(t)^{\frac{1}{(p+1)}}} \in L^{p+1} \underbrace{\left(\varepsilon(t) \int_{w} u_{t}^{2} \alpha(x, t) d x\right)^{\frac{p}{p+1}}} \in L^{\frac{p+1}{p}} d t \\
\leq & C_{m}\left(\int_{S}^{T} E(t)^{\sigma(p+1)+(1-\theta)} \phi^{\prime}(t) d t\right)^{\frac{1}{(p+1)}} \cdot\left(\int_{S}^{T} \varepsilon(t) \int_{w} u_{t}^{2} \alpha(x, t) d x d t\right)^{\frac{p}{p+1}} \\
\leq & C_{m} \frac{\epsilon}{p+1} \int_{S}^{T} E(t)^{\sigma(p+1)+(1-\theta)} \phi^{\prime}(t) d t+C_{m} \frac{p}{(p+1) \epsilon} \int_{S}^{T} \varepsilon(t) \int_{w} u_{t}^{2} \alpha(x, t) d x d t .
\end{aligned}
$$

$\sigma$ is defined such that

$$
\sigma(p+1)+(1-\theta)=\sigma+1, \quad \text { that is } \quad \sigma=\frac{\theta}{p}=\frac{N}{2 m p} .
$$

From (5.7) and (6.7) we can deduce: if $\epsilon$ is little enough, there exists a positive constant $C$ such that

$$
\int_{S}^{T} E(t)^{1+\sigma} \phi^{\prime}(t) d t \leq C E(S)^{\sigma+1}+C \int_{S}^{T} \varepsilon(t) \int_{w} u_{t}^{2} \alpha(x, t) d x d t
$$


Now, choosing $\rho$ and $\phi$ carefully, we will estimate the last term of (6.9).

The Choice of the function $\rho$.

Let us assume that $\phi$ is a concave and strictly increasing $C^{2}$ function such that

$$
\phi(t) \longrightarrow+\infty \quad \text { and } \quad \phi^{\prime}(t) \longrightarrow 0 \quad \text { as } t \rightarrow+\infty
$$

Lemma 6.2. If $b$ satisfies (2.6), then there exists $C>0$ such that

$$
\int_{\frac{R}{2}}^{R} \frac{1}{\tilde{\alpha}(r, t)^{p}} d r \leq C \frac{\rho(t)}{b(\rho(t))^{p}} .
$$

Proof.- If $b$ satisfies (2.6), then

$$
\begin{aligned}
\int_{\frac{R}{2}}^{R} \frac{1}{\tilde{\alpha}(r, t)^{p}} d r & =\int_{\frac{R}{2}}^{R-\rho(t)} \frac{1}{\tilde{\alpha}(r, t)^{p}} d r+\int_{R-\rho(t)}^{R} \frac{1}{\tilde{\alpha}(r, t)^{p}} d r \\
& =\int_{\frac{R}{2}}^{R-\rho(t)} \frac{1}{\tilde{a}(r)^{p}} d r+\int_{R-\rho(t)}^{R} \frac{1}{\tilde{a}(R-\rho(t))^{p}} d r \\
& =\int_{\rho(t)}^{\frac{R}{2}} \frac{1}{\tilde{a}(R-r)^{p}} d r+\int_{R-\rho(t)}^{R} \frac{1}{\tilde{a}(R-\rho(t))^{p}} d r \\
& =\int_{\rho(t)}^{\frac{R}{2}} \frac{1}{b(r)^{p}} d r+\int_{R-\rho(t)}^{R} \frac{1}{b(\rho(t))^{p}} d r \\
& \leq C \frac{\rho(t)}{b(\rho(t))^{p}}+\frac{\rho(t)}{b(\rho(t))^{p}} .
\end{aligned}
$$

Using (6.11) we obtain the following estimation for $\varepsilon$.

$$
\varepsilon(t) \leq \phi^{\prime}(t) \frac{\rho(t)^{\frac{1}{p}}}{b(\rho(t))} .
$$

Since $b$ is strictly increasing near to 0 , we define $\rho$ :

$$
\rho(t):=b^{-1}\left(\phi^{\prime}(t)\right) .
$$

We observe that $\rho$ is decreasing, since $b$ is increasing and $\phi^{\prime}$ is decreasing. From definition of $\rho$ and (6.12) we have

$$
\varepsilon(t) \leq C \rho(t)^{\frac{1}{p}} .
$$


Also, we obtain

$$
\begin{aligned}
\int_{w} \alpha(x, t) u_{t}^{2} d x & =\int_{\frac{R}{2} \leq|x| \leq R-\rho(t)} \alpha(x, t) u_{t}^{2} d x+\int_{|x|>R-\rho(t)} \alpha(x, t) u_{t}^{2} d x \\
& \leq \int_{\Omega} a(x) u_{t}^{2} d x+\int_{|x|>R-\rho(t)} \tilde{a}(R-\rho(t)) u_{t}^{2} d x \\
& \leq-E^{\prime}(t)+b(\rho(t)) E(t) \\
& =-E^{\prime}(t)+\phi^{\prime}(t) E(t) .
\end{aligned}
$$

From (6.9), using $(6.15),(6.14), \rho(t)^{\frac{1}{p}} \leq \rho(S)^{\frac{1}{p}}$ and the fact that $E$ decreases, we have

$$
\begin{aligned}
& \int_{S}^{T} E(t)^{\sigma+1} \phi^{\prime}(t) d t \\
\leq & C E(S)^{1+\sigma}+C \int_{S}^{T} \varepsilon(t)\left(-E^{\prime}(t)+\phi^{\prime}(t) E(t)\right) d t \\
\leq & C E(S)^{1+\sigma}+C \int_{S}^{T} \rho(S)^{\frac{1}{p}}\left(-E^{\prime}(t)\right) d t+C \int_{S}^{T} \rho(t)^{\frac{1}{p}} \phi^{\prime}(t) E(S) d t \\
\leq & C E(S)^{1+\sigma}+C \rho(S)^{\frac{1}{p}}\{E(S)-E(T)\}+C E(S) \int_{S}^{T} \rho(t)^{\frac{1}{p}} \phi^{\prime}(t) d t \\
(6.16 \leq & C E(S)^{1+\sigma}+C \rho(S)^{\frac{1}{p}} E(S)+C E(S) \int_{S}^{T} \rho(t)^{\frac{1}{p}} \phi^{\prime}(t) d t .
\end{aligned}
$$

\section{The choice of the function $\phi$.}

Here, we will show how to define $\phi$ such that $\int_{1}^{+\infty} \rho(t)^{\frac{1}{p}} \phi^{\prime}(t) d t$ is finite.

Let $p^{\prime}>1+p$ and define

$$
\psi(t):=1+\int_{1}^{t} \frac{1}{b\left(\frac{1}{r^{p^{\prime}}}\right)} d r, \quad \forall t>1
$$

Then, $\psi$ is an strictly increasing, convex and $C^{2}$ function, which satisfies

$$
\psi(t) \longrightarrow+\infty \quad \text { and } \quad \psi^{\prime}(t)=\frac{1}{b\left(\frac{1}{t^{p^{\prime}}}\right)} \longrightarrow+\infty \quad \text { as } t \rightarrow+\infty
$$

Define

$$
\phi(t):=\psi^{-1}(t), \quad \forall t \geq 1 .
$$

Then $\phi$ is a strictly increasing, concave and $C^{2}$ function, satisfying $(6.19) \phi(t) \longrightarrow+\infty \quad$ and $\quad \phi^{\prime}(\psi(t))=\frac{1}{\psi^{\prime}(t)} \rightarrow 0 \quad$ as $\quad t \rightarrow+\infty$. 
Thus $\phi$ has all the properties that we use to get (5.7) and (6.16). Using $\rho(t)=b^{-1}\left(\phi^{\prime}(t)\right)$ and making a change of variable $t=\psi(\tau)$, using (6.19) and making another change of variable $\tau=\phi(t)$, and using $b^{-1}\left(\frac{1}{\psi^{\prime}(\tau)}\right)=$ $b^{-1}\left(b\left(\frac{1}{\tau^{p^{\prime}}}\right)\right)=b^{-1} \circ b\left(\frac{1}{\tau^{p}}\right)=\tau^{-p^{\prime}}$ we obtain

$$
\begin{aligned}
\int_{1}^{+\infty} \rho(t)^{\frac{1}{p}} \phi^{\prime}(t) d t & =\int_{1}^{+\infty}\left[b^{-1}\left(\phi^{\prime}(t)\right)\right]^{\frac{1}{p}} \phi^{\prime}(t) d t \\
& =\int_{1}^{+\infty}\left[b^{-1}\left(\phi^{\prime}(\psi(\tau))\right)\right]^{\frac{1}{p}} d \tau \\
& =\int_{1}^{+\infty}\left[b^{-1}\left(\frac{1}{\psi^{\prime}(\tau)}\right)\right]^{\frac{1}{p}} d \tau \\
& =\int_{1}^{+\infty} \frac{1}{\tau^{\frac{p^{\prime}}{p}}} d \tau \\
& =\lim _{M \rightarrow+\infty} \frac{1}{-\frac{p^{\prime}}{p}+1}\left\{M^{-\frac{p^{\prime}}{p}+1}-1\right\} \text { and since }-\frac{p^{\prime}}{p}+1<0 \\
& =\frac{1}{\frac{p^{\prime}}{p}-1}>0 .
\end{aligned}
$$

Estimation depending on $\phi$.

Since $\psi \circ \phi=I$ then $\psi^{\prime}(\phi(t)) \phi^{\prime}(t)=1$, and then $\phi^{\prime}(t)=\frac{1}{\psi^{\prime}(\phi(t))}=$ $b\left(\frac{1}{\phi(t)^{p^{\prime}}}\right)$, from which we deduce

$$
\rho(t)=b^{-1}\left(\phi^{\prime}(t)\right)=b^{-1} \circ b\left(\frac{1}{\phi(t)^{p^{\prime}}}\right)=\frac{1}{\phi(t)^{p^{\prime}}} .
$$

By other hand, using (6.20), we obtain

$$
\begin{aligned}
\int_{S}^{T} \rho(t)^{\frac{1}{p}} \phi^{\prime}(t) d t & =\int_{S}^{T} \frac{1}{\phi(t)^{\frac{p^{\prime}}{p}}} d t \\
& =\frac{1}{1-\frac{p^{\prime}}{p}}\left[\phi(t)^{1-\frac{p^{\prime}}{p}}\right]_{S}^{T} \\
& =\frac{1}{1-\frac{p^{\prime}}{p}}\left\{\phi(T)^{1-\frac{p^{\prime}}{p}}-\phi(S)^{1-\frac{p^{\prime}}{p}}\right\} \\
& =\frac{1}{\frac{p^{\prime}}{p}-1}\left\{\phi(S)^{1-\frac{p^{\prime}}{p}}-\phi(T)^{1-\frac{p^{\prime}}{p}}\right\} \\
& \leq \frac{1}{\frac{p^{\prime}}{p}-1}\left\{\phi(S)^{1-\frac{p^{\prime}}{p}}\right\} \quad \text { since } \phi=\psi^{-1}>0 .
\end{aligned}
$$


Using (6.20) and (6.21) in (6.16) we have

$$
\begin{aligned}
\int_{S}^{T} E(t)^{\sigma+1} \phi^{\prime}(t) d t & \leq C E(S)^{1+\sigma}+E(S) \frac{C}{\phi(S)^{\frac{p^{\prime}}{p}}}+E(S) \frac{C}{\phi(S)^{\frac{p^{\prime}}{p}-1}} \\
& \leq C E(S)^{1+\sigma}+E(S) \frac{C}{\phi(S)^{\frac{p^{\prime}}{p}}-1} .
\end{aligned}
$$

Then apply Lemma 4.4 since (4.26) holds with $\sigma^{\prime}=\frac{p^{\prime}}{p}-1>0$, and deduce that exists a constant $C$ depending continuously on $E(1)$, such that

$$
E(t) \leq \frac{C}{\phi(t)^{\frac{p^{\prime}}{p \sigma}}}=\frac{C}{\phi(t)^{\frac{p^{\prime}}{\theta}}}, \quad \forall t \geq 1 .
$$

\section{Growth of $\phi$.}

Estimate the growth of $\phi$ is equivalent to bound the function $\phi^{-1}=\psi$.

Let $T_{0}$ such that $b\left(\frac{1}{\tau^{p^{\prime}}}\right) \leq 1, \quad \forall \tau \geq T_{0}$.

If $s<\tau$ and since $b$ is increasing we have $b\left(\frac{1}{\tau^{p^{\prime}}}\right) \leq b\left(\frac{1}{s^{p^{\prime}}}\right)$, i.e. $\frac{1}{b\left(\frac{1}{s p^{\prime}}\right)} \leq$ $\frac{1}{b\left(\frac{1}{\tau p^{\prime}}\right)}$.

On other hand, we have that: if $p^{\prime} \geq 1,1+(\tau-1) z \leq \tau^{p^{\prime}} z$ holds for $z \geq 1$ and $\tau \geq 1$. In fact, we only have to prove that $1 \leq\left(1-\tau+\tau^{p^{\prime}}\right) z$. If $\tau \geq 1$ then $\tau^{p^{\prime}}-\tau \geq 0$ and so $1+\tau^{p^{\prime}}-\tau \geq 1$; then multiplying by $z \geq 1$ we have $\left(1-\tau+\tau^{p^{\prime}}\right) z \geq 1$. Using these remarks we obtain

$$
\begin{aligned}
\psi(\tau) & =1+\int_{1}^{\tau} \frac{1}{b\left(\frac{1}{s^{p}}\right)} d s \leq 1+\frac{1}{b\left(\frac{1}{\tau^{p^{p}}}\right)} \int_{1}^{\tau} d s \leq 1+(\tau-1) \frac{1}{b\left(\frac{1}{\tau^{p^{\prime}}}\right)} \\
& \leq \tau^{p^{\prime}} \cdot \frac{1}{b\left(\frac{1}{\tau^{p^{\prime}}}\right)}=\frac{1}{B\left(\frac{1}{\tau^{p^{\prime}}}\right)} .
\end{aligned}
$$

Then, letting $t=\frac{1}{B\left(\frac{1}{\tau^{p}}\right)}$ ( that is $\left.\frac{1}{\tau^{p^{\prime}}}=B^{-1}\left(\frac{1}{t}\right)\right)$ and using (6.24) we have $\psi(\tau) \leq t$, from which $\tau \leq \psi^{-1}(t)=\phi(t)$, that is

$$
\frac{1}{\phi(t)} \leq \frac{1}{\tau} \text {. }
$$

Thus, using (6.25) in (6.23) we obtain

$$
E(t) \leq \frac{C}{\phi(t)^{\frac{p^{\prime}}{\theta}}} \leq \frac{1}{\tau^{\frac{p^{\prime}}{\theta}}}=\left(B^{-1}\left(\frac{1}{t}\right)\right)^{\frac{1}{\theta}},
$$

where $\theta=\frac{N}{2 m}$. 


\section{Acknowledgements}

Y.S.S.A was partially suppoted by C.S.I. Estudio $\mathrm{N}^{\circ} 06140104$, Universidad Nacional Mayor de San Marcos (UNMSM-Lima, Perú). The professor L.C.V was partially supported by the Chile Science Foundation Conicyt-Fondecyt Grant 1040067. Also, he was partially supported by a Internal project of the Universidad de Antofagasta (UA- Antofagasta, Chile), DIRINV Grant 1319-06 and a Grant of CNPq, PROSUL, (Brasil) "Programa Sul-Americano de Apoio às Atividades de Cooperação em Ciência e Tecnologia", respectively.

\section{References}

[1] F. Conrad and B. Rao - Decay of solutions of wave equations in a star-shaped domain with nonlinear boundary feedback. Asympt. Anal. 7, pp. 159-177, (1993).

[2] L. Cortés-Vega.- A note on resonant frequencies for a system of elastic wave equations, Int. J. Math. Math. Sci. 64, pp. 3485-3498, (2004).

[3] L. Cortés-Vega and Y. Santiago-Ayala, Decaimiento de la ecuación de onda con disipación. PESQUIMAT Revista de la Fac. CC. MM. de la UNMSM. Vol IX, Nro. 1, pp. 39-62, (2006).

[4] L. Hörmander - Linear Partial Differential Operators, Springer-Verlag, New York, 116, (1976).

[5] M. Ikawa - Mixed problems for hyperbolic equations of second order. J. Math. Soc. Japan 20, pp. 580-608, (1968).

[6] S. Kesavan - Topics in Functional Analysis and applications. John Wiley \& Sons, (1989).

[7] J. Lagnese, Deacay of solutions of wave equations in a bounded region with boundary dissipation, J. Differential Equations 50, pp. 163-182, (1983).

[8] J. Lagnese and J. L. Lions, Modelling Analysis and Control of thin Plates, Masson, Paris, (1989). 
[9] I. Lasiecka and R. Triggiani, Control Problems for Systems Described by Partial Differential Equations and Applications, Springer Verlag Lect. Not., 97 (1987).

[10] I. Lasiecka and G. Avalos, Uniform decay rates of solutions to a structural acoustic model with nonlinear dissipation, Appl. Math Comp. Sci, 8, No. 2, pp. 101-127, (1998).

[11] I. Lasiecka and D. Tataru, Uniform boundary stabilization of semilinear wave equations with nonlinear boundary damping, Diff. Int. Eq, 6, pp. 507-533, (1993).

[12] I. Lasiecka, Stabilization of wave and plate-like equations with nonlinear dissipation on the boundary, J. Diffetential Equations, 79, pp. 340-381, (1989).

[13] V. Komornik- Exact controllability and stabilization. John Wiley \& Sons, (1994).

[14] V. Komornik and E. Zuazua, A direct method for the boundary stabilization of the wave equation, J. Math. Pures Appl. 69, pp. 163-182, (1990).

[15] P. Martinez. - Decay of solutions of the wave equation with a local highly degenerate dissipation. Asymptotic Analysis 19, pp. 1-17, (1999).

[16] M. Nakao - Decay of solutions of the wave equation with a local degenerate dissipation. Israel J. of Maths 95, pp. 25-42, (1996).

[17] A. Pazy - Semigroups of Linear Operators and Applications to Partial Differential Equations. Springer, New York, (1983).

[18] Y. Santiago A. - Una aplicación del Lema de Nakao. PESQUIMAT Revista de la Fac. CC. MM. de la UNMSM, Nro. 2, pp. 23-36, (2006).

[19] Y. Santiago A. - Decaimiento exponencial de la solución débil de una ecuación de onda no lineal. PESQUIMAT Revista de la Fac. CC. MM. de la UNMSM. Vol VIII Nro. 2, pp. 29-43, (2005).

[20] Y. Santiago A. and J. Rivera - Global existence and exponential decay to the wave equation with localized frictional damping. PESQUIMAT Revista de la Fac. CC. MM. de la UNMSM. Vol V. Nro. 2, pp. 1-19, (2002). 
[21] D. Tataru, Boundary controllability for conservative PDEs, Appl. Math. Optim. 3, pp. 257-295, (1995).

[22] E. Zuazua - Exponential decay for the semi-linear wave equation with locally distributed damping. Comm. Partial Differential Equations. 15, pp. 205-235, (1990).

\section{Luis A. Cortés-Vega}

Departamento de matemáticas

Universidad de Antofagasta

Facultad de Ciencias Básicas

Casilla 170

Chile

email : lcortes@uantof.cl

and

Yolanda S. Santiago-Ayala

Facultad de Ciencias Matemáticas

Universidad Nacional Mayor de San Marcos

Ciudad Universitaria

Avenida Venezuela, cdra. 34

Lima 1

Perú

e-mail : ysantiagoa@unmsm.edu.pe 